\title{
Ultrasensitive and towards single molecule SERS: general discussion
}

Javier Aizpurua, (D) Heike Arnolds, Jeremy Baumberg, lan Bruzas, Rohit Chikkaraddy, Malama Chisanga, Paul Dawson, (ID Volker Deckert, (D) Ines Delfino, (D) Bart de Nijs, (D) Giuliana Di Martino, (iD Joshua Edel, Holly Fleming, Sylwester Gawinkowski, (DD Fabrizio Giorgis, Roy Goodacre, Duncan Graham, (iD Mike Hardy, (ID Christian Heck, (D) Sebastian Heeg, Kevin Hewitt, Lauren Jamieson, Alex Keeler, Agata Królikowska, Christian Kuttner, Nathalie Lidgi-Guigui, Carin Lightner, John Lombardi, Sumeet Mahajan, Natalia Martín Sabanés, (iD Jean-Francois Masson, (iD Niclas Sven Mueller, (D) Howbeer Muhamadali, Kei Murakoshi, Jürgen Popp, Marc Porter, (iD Stephanie Reich, George Schatz, Zhong-Qun Tian, Ashish Tripathi, Richard Van Duyne, Xinping Wang, Alastair Wark, Katherine (Kallie) Willets and Marjorie Willner (ID

\section{DOI: $10.1039 / C 7 F D 90088 K$}

Ashish Tripathi opened a discussion of the paper by Volker Deckert: TERS imaging of the triangular shaped nanoparticle with a D3ATP monolayer is shown in Fig. 3 of your paper. ${ }^{1}$ The chemical map of the $1438 \mathrm{~cm}^{-1}$ band of the D3ATP monolayer suggests that the signal intensities are higher at the edges than near the centre (Fig. 3, bottom left). Can you comment?

1 Z. Zhang, M. Richard-Lacroix and V. Deckert, Faraday Discuss., 2017, DOI: 10.1039/ c7fd00157f.

Volker Deckert responded: Certainly an additional SERS effect caused by the edges and the nanoscale defects would influence the observed intensity and those effects can even be expected. With respect to the experiments we usually try to stay away from the edges in order to avoid potential effects at the edges. In general we would be cautious here not to over-interpret the situation at the edges, as the edges might also be favoured locations for sample aggregation. And both effects, higher concentration and field enhancement, would result in an apparent signal increase, but are difficult to distinguish. Hence, we generally stay away from the actual edge.

Jürgen Popp asked: The experiments were performed in the transmission mode. How does the laser polarization affect the TERS spectra? 
Volker Deckert answered: The laser polarisation in fact influences the TERS spectra considerably. The experiment resembles, in a way, a single crystal Raman experiment with a fixed geometry. In addition the tip acts as an antenna which is also strongly dependent on the polarisation. For a more detailed overview we suggest having a look at "Mastering high resolution tip-enhanced Raman spectroscopy: towards a shift of perception". ${ }^{1}$

1 M. Richard-Lacroix, Y. Zhang, Z. Dong and V. Deckert, Chem. Soc. Rev., 2017, 46, 39223944.

Marc Porter asked: Can you help me understand the data in Fig. 4 of your paper? I am having trouble understanding the spectral data in terms of the signal strength and how the noise levels differ for the four different spectra. The noise level for the $60 \mathrm{~s}$ laser exposure is lower than those for the other three spectra. This difference points to a difference in the amplitudes of the spectral features for the spectrum at $60 \mathrm{~s}$ with respect to the other three spectra. Can you comment on the origin of these differences?

Volker Deckert answered: Before trying to answer the question, some background information: all spectra we show, including Fig. 7 are raw untreated data that indicate the realistic noise levels that occur during our TERS experiments. We are aware that the experimental conditions can influence the absolute intensities of the Raman spectra considerably. Therefore we used only intensity ratios and frequency positions for the evaluation. This way we ensure that the spectra measured at different time slots can be still compared. Nevertheless, the question regarding the different noise levels is valid. In Fig. 7 the SNR difference between $40 \mathrm{~s}$ and $60 \mathrm{~s}$ could be easily explained by the $5 \times$ higher laser intensity. However, this does not explain why the $90 \mathrm{~s}$ and $120 \mathrm{~s}$ spectra then show a much lower SNR again. If we compare this with previous experiments using Ag-TERS tips (e.g. P. Singh et al. ${ }^{1}$ ) we see that this phenomenon is quite pronounced when working under ambient conditions but not when working under inert gas conditions. Consequently, we attributed this behaviour to a slow tip degradation (oxidation). So most likely we face the same behaviour here, emphasising the importance of the aforementioned evaluation of the relative intensities only.

\section{P. Singh and V. Deckert, Chem. Commun., 2014, 50, 11204-11207.}

Stephanie Reich asked: Different reaction mechanisms may be important for the polymerization in your system, e.g. photochemical, hot electron induced, or thermal. How important do you think the respective mechanisms are? Do you have an experimental tool to distinguish between them?

Volker Deckert responded: This is a very interesting question. We know from previous studies that the reaction occurs under illumination with green light alone (see ref. 1). Thus, we cannot (and did not) exclude that the reaction is partially photo-activated. It was however also observed that the kinetics of such a reaction in the bulk are much slower compared to the reaction under plasmonic conditions. As stated in the introduction of our manuscript, "plasmonic conditions" does not only mean "hot electrons". The enhancement of the electric field alone can potentially induce a photo-activated reaction. Thus, in a situation where 
both hot electron and photo-induction are likely to be implied, one can hardly distinguish the respective contributions. For this purpose investigations in an inert atmosphere are required as in ref. 1 . In theory, one could indirectly extract the bulk and strictly photo-activated reaction kinetics from the global plasmonic effect using time resolved experiments. Unfortunately, the reaction is so fast that even at very low incident laser power, the first spectrum (measured after $1 \mathrm{~s}$ ) already shows all the characteristic features of the reaction product and as such this is beyond the current capabilities of most TERS setups.

1 Z. Zhang, D. Kinzel and V. Deckert, J. Phys. Chem. C, 2016, 120, 20978-20983.

Roy Goodacre said: The main spectral changes you observe have really just been eyeballed and I wondered if you had considered chemometric methods like multivariate curve resolution (MCR), which can perform spectral deconvolution? i.e., to use mathematical approaches that can "unmix" these spectra into pure component spectra and their concentrations.

Volker Deckert responded: This is an excellent suggestion and collaborations here are very welcome. The main reason for the presented evaluation of the peaks was the fact that the signals could be easily distinguished and assigned. The suggested unmixing would certainly help to unravel the origin of the different species. It might however be challenging to obtain reference data for the pure components.

Roy Goodacre continued: As a follow-up - and this depends on the concentration of the reaction product - most of this work is based purely on the interpretation of TERS spectra. I wonder whether you think you can use orthogonal analytical techniques like liquid chromatography with mass spectrometry or MS directly to identify products and any intermediates with better chemical specificity, and also to calculate reaction kinetics?

Volker Deckert answered: It would be interesting to corroborate the results with a second technique. However, one must keep in mind that these are monolayers linked by a sulphide bond with the gold surface. In TERS especially, the reaction is induced on a single plate. Thus, there are two challenges:

(1) The molecules should be detached from this same single gold plate (without destroying the polymer).

(2) Even if this was possible, the technique sensitivity is not high enough to quantitatively detect so few molecules (if considering MS alone).

The reaction is so quick that no intermediate product could be identified by vibrational spectroscopy. Since the reaction happens at the scale of a few molecules under the tip, a small fraction of side product or reaction intermediates would likely be detected by TERS if their life time was long enough. As such, it would be extremely challenging to stop the reaction at a given time (that is fairly small) and homogeneously over the plate area. Finally, since we are forming a polymer, its length (or molecular weight) could prevent vaporisation.

At this time, we do not see a second independent technique giving access simultaneously to high spatial resolution, high sensitivity and high chemical specificity. One of the possible avenues we are currently exploring is the corroboration of chemical and local mechanical property changes, measured with the 
use of AFM. Since the reacting groups are in the plane of the substrate for this polymer, it is unlikely to produce notable differences. However, this strategy could be used for other reactions.

Duncan Graham asked: What about cis-trans isomerisation of the azo linkage? Even on a metal surface, you won't have all of it as an S-metal bond. Could you get disulphide linkages formed that affect the conformation and hence the spectra?

Volker Deckert answered: Indeed, we have considered a cis to trans conformational change, but we have eliminated this possibility because the cis conformation would give rise to a strong band associated with the azo group at approximately $1500 \mathrm{~cm}^{-1}$ ( $\mathrm{N}=\mathrm{N}$ elongation) (see ref. 1). If, for instance, the $1530 \mathrm{~cm}^{-1}$ band was somewhat associated with this type of conformational change, it would also cause a decrease of the $1438 / 1575 \mathrm{~cm}^{-1}$ band ratio with time. The cis or trans conformations are both consistent with all the thio groups being bonded to the metal surface because those conformations can be in the plane of the substrate (see the molecular structure in Scheme 1 of our paper). In the TERS experiment, the disulfide band appears in the region of the silicon band arising from the silver coated AFM tip (typically at $\sim 500 \mathrm{~cm}^{-1}$ ) and is thus difficult to use for evaluation. In our SERS experiment (with no silicon interference), this region was free of any band. Thus we concluded that all the probed molecules (or the vast majority) had two Au$\mathrm{S}$ bonds. This does not imply that the system is not dynamic. The diffusion of the gold atoms at the surface and the deformation/reformation of the S-metal bond are expected to enable the molecular reorganization necessary for all the molecules to react and adopt the most thermodynamically stable conformation.

1 Y. F. Huang, H. P. Zhu, G. K. Liu, D. Y. Wu, B. Ren and Z. Q. Tian, J. Am. Chem. Soc., 2010, 132, 9244-9246.

Joshua Edel enquired: What is the correlation between the reaction yields, purities/side product formation and field enhancement?

Volker Deckert answered: This is a very good question and tough to answer. Part of the difficulty in evaluating the yield and purity aspects is related to the fact that the laser illumination is used both for inducing the reaction and for probing. Since the kinetics of the reaction are fast under plasmonic conditions, no matter where the tip is located, the spectra will show the strong bands of the product. Additionally, we do not have well isolated bands that could help in probing the disappearance of the $\mathrm{NH}_{2}$ group in the spectral region investigated, which further complicates the analysis. We thus rely exclusively on the net shift of the $1590 \mathrm{~cm}^{-1}$ band (the $\mathrm{C}=\mathrm{C}$ stretching of the initial molecule) to $1575 \mathrm{~cm}^{-1}$ when the azo group is formed. In Fig. 5 of our paper, we have shown the relative intensity evolution of the $1438 \mathrm{~cm}^{-1}$ band (the $\mathrm{N}=\mathrm{N}$ elongation mode) with respect to the $1575 \mathrm{~cm}^{-1}$ band (or $1150 \mathrm{~cm}^{-1}$ band). The plateauing of this intensity ratio after a few seconds of light exposure is a indirect indication of the completion of the reaction and the "purity" of the product formed. However, this is true only for "everything" under the tip and is consequently limited to a few molecules. This brings up an even more fundamental question: how do we define the yield in a TERS tip induced reaction? If we discuss only the fraction directly in 
the probed region ("under the tip"), then we can approximate a yield close to $100 \%$. If we consider the entire monolayer of a single plate, this depends on the methodology employed to induce the reaction at this larger scale.

Agata Królikowska asked: You reported the appearance of two TERS peaks of unknown origin, and at least one of them was taken as a signature of polymerization.

Going back to the tautomers, if the molecule is attached by only one thiol group (one detached by the tip or for some other reason), which the undergoes a deprotonation and this proton migrates, then we can speculate that an elimination of an ammonia molecule takes place. This would lead to a biphenyl molecule with one thiolate, and one thione group, monosubsituted by an amino group. Have you considered that the observed TERS changes may be due to this or a similar tip/plasmon induced deprotonation reaction instead of polymerization?

Volker Deckert responded: We have indeed considered the possibility of a thioketone formation, especially since it would lead to a band in the $\sim 1200$ to $1275 \mathrm{~cm}^{-1}$ region that could justify the $1260 \mathrm{~cm}^{-1}$ strong band observed in TERS after scanning (see Fig. 8 of the article). This is not directly mentioned, but rather implied in the proposed "photo-degradation process" that is ruled out in the article. In fact, it is unlikely to be simply a plasmon-induced deprotonation since the molecule is initially linked to the metal. A S-Au bond breakage is required, followed by the protonation and deprotonation of the same group. In short, no matter by which mechanism this species could have been formed, it is expected to be by a multiple step reaction. We have eliminated this possibility primarily by considering the comparison of the TERS single point $v s$. TERS after pre-scanning results. The $1260 \mathrm{~cm}^{-1}$ band is absent from the time-resolved TERS, where the molecules have experienced a longer exposure to light and under plasmonic conditions. In this latter context, the molecule would have been more likely to undergo such a complex mechanism (that implies slower kinetics). We have also considered that the expulsion of an ammonia molecule could be indirectly tracked by the reduction of the intensity of the azo group-related bands with respect to the 1150 or $1575 \mathrm{~cm}^{-1}$ band (see Fig. 5 of the article). In contrast, we observed a splitting of those bands, suggesting that the azo population remains constant but conformation changes happen (causing a change of the band position). One must also keep in mind that the formation of a thioketone implies loss of the aromaticity of the phenyl group on which it is situated. This should cause a shift (or a split) of the $1575 \mathrm{~cm}^{-1}$ band to lower wavenumbers that we have not observed. In fact, one could argue that it could justify the presence of the $1530 \mathrm{~cm}^{-1}$ band. On the other hand, the $1530 \mathrm{~cm}^{-1}$ band has been shown to be present in all the SERS and TERS experiments while the $1260^{-1}$ did not appear.

Nathalie Lidgi-Guigui commented: In the conclusions of your paper, you say "most likely a thermally controlled second reaction takes place giving rise to the Raman signal at $1530 \mathrm{~cm}^{-1}$ ". Yet you said that you saw this peak at room temperature, so do you have any idea of the process behind this second reaction?

Volker Deckert replied: By "thermally controlled", we intended that there is a thermodynamically driven second step, this does not necessarily require "high" 
temperatures. As stated above, the temperature felt by the molecule in the near field is a complex topic, but is expected to differ slightly from room temperature. Our hypothesis is that once a few monomers are connected, the biphenyl groups reorganize with respect to each other to reach a more stable and potentially less strained relative orientation/organization. This process is slower than the coupling reaction and leads to a more thermodynamically stable conformational distribution. We would not consider this as a chemical reaction in the normal sense. It only requires a steric rearrangement. Thus, our hypothesis is that the polymer is initially kinetically trapped due to the fast coupling reaction, grows, and reorganizes its conformation with time.

Jeremy Baumberg commented: You see the clear appearance of new lines at $1260 \mathrm{~cm}^{-1}$ but only when you do some sort of long-term scanning, not at a single point. Have you tried spatially mapping the sample area afterwards, to see if you can see the limits of this conversion spatially? Is there a confined region within which you excite, or is it in effect a much larger region since the polymer initiation can propagate? Can you irradiate a narrow line instead to see how far the conversion can propagate?

Volker Deckert replied: We have not tried to spatially map the $1260 \mathrm{~cm}^{-1}$ band after scanning, but this is indeed a good suggestion that could strengthen the argument.

We cannot exclude completely that the polymerization may propagate. However, comparison of the spectra from point scanning (time-dependent TERS at a single point) and TERS after scanning under illumination strongly suggests that it does not happen, or that this is not the main mechanism. If the reaction propagates, for instance, from radical formation (from a single electron transfer from the tip to the molecule), the results should be identical for both types of experiment and be even more visible over the minute time scale of the single point experiment. The radical would not follow a straight line trajectory and would rather go in all the directions of space where it could find a group to react with. Therefore, following a line scan to track the scale at which it happens may not be the best way. We would like to emphasize that the fact that the reaction is induced by the same probe that is used for detection is problematic for any attempt at deconvolution, especially when the kinetics are as fast as they are here. Ideally this could be separated (e.g. as distinct wavelengths).

Nathalie Lidgi-Guigui remarked: My comment addresses a question about the influence of light polarization when taking advantage of plasmons to functionalize nanoparticles.

I can share some data that can be found in detail in the last issue of Nanoscale Horizons, in a communication titled "Multi-functionalization of lithographically designed gold nanodisks by plasmon-mediated reduction of aryl diazonium salts". ${ }^{1}$

1 I. Tijunelyte, I. Kherbouche, S. Gam-Derouich, M. Nguyen, N. Lidgi-Guigui, M. Lamy de la Chapelle, A. Lamouri, G. Lévi, J. Aubard, A. Chevillot-Biraud, C. Mangeney and N. Felidj, Nanoscale Horiz., 2017, DOI: 10.1039/c7nh00113d.

Zhong-Qun Tian commented: TERS is an excellent tool, especially with its extremely high spatial resolution. However, the amount of probed species under 
the TERS tip is very small, making the overall sensitivity (overall scattered light) quite low. One example is pyridine as one of the "star" and commonly used molecules in SERS. So far the TERS of pyridine has not yet been reported mainly because the adsorption of pyridine is not strong and these molecules are quite mobile in and out from the surface under the tip. It may be noticed that the molecules will have surface diffusion and/or surface desorption from the surface to the solution/gas phase. In general TERS and SERS both face problems studying weakly adsorbed species, but the situation for TERS is worse due to its lower sensitivity. TERS has been restricted so far to those surface molecules that are not very mobile. Accordingly I wonder how far TERS can go regarding the study of surface reactions. It's not easy to find systems where both the reactant and product can bind to the probed surface, which will make the reaction yield and turn-over rate extremely low. Moreover, to get better sensitivity, TERS must utilise a plasmonic substrate, e.g., Au or Ag, for optimizing the hot spot between the tip and substrate. This means most commonly used substrates for surface catalysis and electrochemistry cannot be directly applied. To in situ monitor the reaction process is indeed very promising but challenging, if one can find a way to improve the versatility significantly. Can we rationally design some special nanostructures for tips to further increase the TERS sensitivity considerably?

Volker Deckert answered: This is a very valuable assessment of the current status of TERS on reacting molecules, however, I think several aspects of this statement need to be clarified. Specifically the argument regarding sensitivity should be rather the other way round. TERS has obviously the sensitivity to detect a "very small" amount of probed molecules. This means that in several cases TERS experiments even show single molecule sensitivity and surely several tens of molecule sensitivity would be considered sufficient. That the overall signal is lower compared to SERS does not matter, as long as the signal strength is sufficient for detection. It is an altogether different question if the entire composition of the surface is the target. But also here one could argue, that the specific binding of molecules, as in SERS, is in general not an issue for TERS as long as the probed molecule does not bind to the probe. With respect to the mentioned pyridine, I can only answer from our groups perspective, that for TERS more reliable ways exist to provide a stable substrate for fundamental experiments - namely SAMs. Regarding the challenges due to surface diffusion the items mentioned are certainly correct. However, to investigate such phenomena we can only use the tools that are available and improve them step-by-step. Already, the UHV cryogenic temperature experiments show a way to address such challenges. Also, functionalising a molecule such that it binds/adsorbs to a surface is fine as long as the limitations of this approach are understood (No one would question fluorescent labelling experiments because an artificial molecule is introduced into a system). Last but not least, if the mentioned diffusion process needs to be addressed, eventually a tool that investigates the microscopic behaviour of the system (TERS) could be preferable to a tool that addresses average information (standard SERS). Obviously here TERS and single molecule SERS would ultimately provide the same information.

Another important aspect needs to be emphasized here. It is a common misunderstanding that gap modes are required for sensitive TERS. This is simply not the case. Already the first TERS experiments did not utilise gap modes, and later TERS experiments show high quality TERS spectra of, for instance, single RNA 
strands immobilised on mica. Certainly a gap mode will further enhance the signal, but it is not necessary to sacrifice the generality of a certain sample approach to the need for working on a metallic substrate (and for that matter, it does not have to be $\mathrm{Au}, \mathrm{Ag}$, or $\mathrm{Cu}$ ). To address the last part of the comment - I can only agree. It is challenging and it is important to improve the versatility and the probes. The good news is that many new aspects of the theory of tip sample interaction are just being investigated. In particular for TERS probe design I would expect that new ideas based for instance on the results of Taguchi et al., ${ }^{1}$ will yield more efficient TERS tips. Provided that such tips are sensitive enough one does not need to sacrifice the above mentioned versatility, simply because the probes are sensitive enough.

1 A. Taguchi, J. Yu, P. Verma and S. Kawata, Nanoscale, 2015, 7, 17424-17433.

Joshua Edel opened a discussion of the paper by Kevin Hewitt: What particles are you using? What is their functionality, and do they have a Raman reporter?

Kevin Hewitt replied: They are derived from the Cabot patent. ${ }^{1}$

1 F. Tam, M. E. Piotti and R. G. Freeman, U. S. Pat. 2012/0156491A1, 2012.

Sylwester Gawinkowski asked: In Fig. 3 and 4 in your paper $^{1}$ you have shown spectra registered on two different nanoparticles, S440 and S470. Can you explain why on the second spectrum (Fig. 7) a strong Raman band is located at about $1160 \mathrm{~cm}^{-1}$, although BPA molecules do not have such a Raman band in conventional SERS. What chemical substance is in the S470 nanoparticles?

1 C. L. D. Lee and K. C. Hewitt, Faraday Discuss., 2017, DOI: 10.1039/c7fd00137a.

Kevin Hewitt replied: The chemical species attached are protected by the aforementioned patent by Oxonica/Cabot. What I can say is that they are derivatives of BPA.

Jeremy Baumberg said: You put quite a lot of power on these systems. Are the particles in liquid or on a substrate? You think you're getting your signal from only the dimers, but for these large power densities, are they not sintered together?

For instance, see ref. 1 which shows how the dimers can be very sensitive to this. Have you used small cuvette volumes and then looked at TEM afterwards (as in this paper)?

1 L. O. Herrmann, V. K. Valev, C. Tserkezis, J. S. Bernard, S. Kasera, O. A. Scherman, J. Aizpurua and J. Baumberg, Nat. Commun., 2014, 5, 4568.

Kevin Hewitt answered: Thank you for this very important question.

Our average powers for both the pulsed and CW experiments were about 0.01$0.1 \mathrm{MW} \mathrm{cm}^{-2}$, whereas the experiments described in your paper use powers of

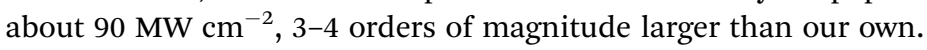

Moreover, in Fig. 5b we show there is no saturation of the SRL with increasing pump or probe laser power, unlike the results of Frontiera et al. ${ }^{1}$ who investigated the same particles that we used, from Oxonica. In both cases the samples are in aqueous solution, at picomolar concentrations in our case. Saturation led 
Frontiera to investigate/propose laser induced damage of the particles as the mechanism, whereas we do not observe such an effect in our experiments.

The difference in our experiments is that we used much lower peak powers: as outlined in Frontiera et al. ${ }^{1}$ the pulsed laser experiments used peak pump powers of $0.1 \mathrm{~W} \mathrm{~cm}^{-2}$ (vs. $0.01 \mathrm{~W} \mathrm{~cm}^{-2}$ for us) and peak probe pulses of $10 \mathrm{~W} \mathrm{~cm}^{-2}$ (versus $0.004 \mathrm{~W} \mathrm{~cm}^{-2}$ for us). Again, we use powers that are orders of magnitude smaller in a very comparable experiment using samples from the same manufacturer, Oxonica.

The signal is also very reproducible, implying a reversible effect, which would not be the case were the samples irreversibly damaged. The solution also offers rapid heat dissipation, and the particles are exchanged within the probe volume readily over the $120 \mathrm{~s}$ collection time.

For all these reasons we are confident that the particles are not damaged.

1 R. R. Frontiera, A.-I. Henry, N. L. Gruenke and R. P. Van Duyne, J. Phys. Chem. Lett., 2011, 2, 1199-1203.

Sumeet Mahajan said: In your SRS result with a CW laser what did you do to confirm that it is indeed SRS? Did you have the pump and Stokes cross-polarised to see whether you still had emission/loss/gain?

Kevin Hewitt answered: CW SRL was confirmed by scanning the frequency difference through a vibrational mode of two different nanoparticle types (S440 and S470 which contain different organic dyes attached to the embedded AuNPs). We are now conducting experiments using cross-polarized pump and probe beams, and are investigating the power dependence of CW stimulated SERS. Stay tuned! We also conducted CW SRL using neat benzene (see Fig. 1), to reproduce Owyong's seminal results. ${ }^{1}$

1 A. Owyoung and E. D. Jones, Opt. Lett., 1977, 1, 152.

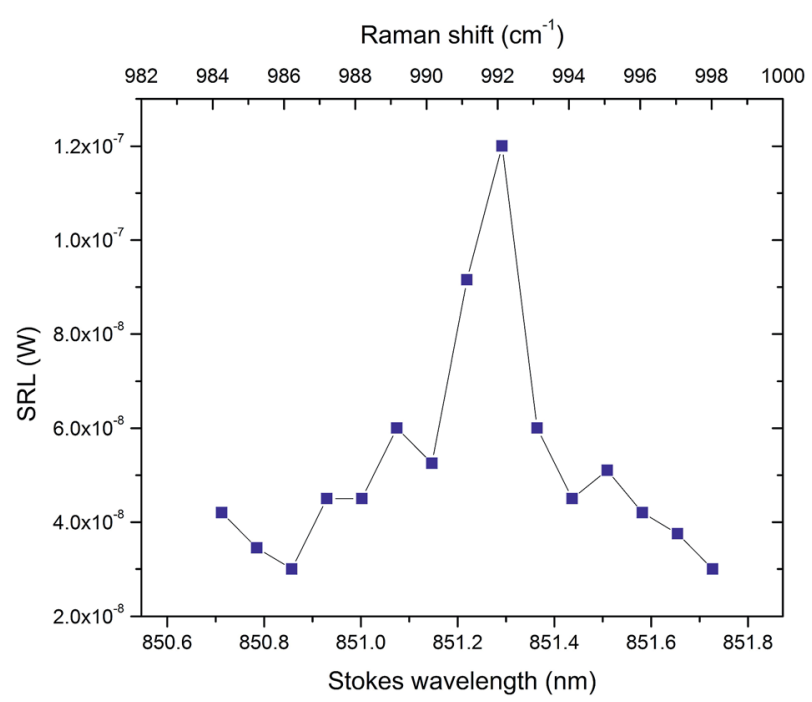

Fig. 1 SRS spectra of neat benzene using CW Ti:sapphire lasers with a $785 \mathrm{~nm}$ pump (100 $\mathrm{mW})$ and probe (100 $\mathrm{mW}, 850.7-851.7 \mathrm{~nm})$. 
Ines Delfino asked: The concentration of the suspension of S-440 nanoparticles is reported in the caption of Fig. 5 of the manuscript as $64 \mathrm{pM}$. What is the concentration of the investigated suspension of S-470 nanoparticles?

Kevin Hewitt responded: Thanks for your question and I apologize for not including it. The concentration is also $64 \mathrm{pM}$

Joshua Edel remarked: In the context of the session topic, I was wondering what concentrations you can reliably probe and what the minimum number of molecules that can be detected is.

Kevin Hewitt replied: I appreciate the question. The signals are generated from sample volumes on the order of $10^{4}$ cubic microns (a cylinder of cross sectional area 1 micron $^{2}$ and a path length of $1 \mathrm{~cm}$ ). With a total (monomer, dimer, trimer) concentration of 64 picomolar, and assuming the signal is generated from dimers, trimers and tetramers which make up $10 \%$ of the sample, we estimate $N=6.4 \mathrm{pM} \times$ Avogadro's number $\times 10^{4}$ cubic microns $\approx 10$ nanoparticles. We therefore estimate that tens of particles and picomolar concentrations can be reliably probed.

Sylwester Gawinkowski said: The bands of your spectra have an ordinary shape similar to normal Raman or SERS spectra, but the dispersive shape of SE-SRS bands was reported by the group of Prof. Richard van Duyne for similar nanoparticles ${ }^{1}$. Could you explain this difference? Is it a result of the different nanoparticle-molecule system or another factor?

1 R. R. Frontiera, A.-I. Henry, N. L. Gruenke and R. P. Van Duyne, J. Phys. Chem. Lett., 2011, 10, 1199-1203.

Kevin Hewitt answered: A dispersive lineshape would produce a node when the frequency difference of the pump and probe matches the frequency of a molecular vibrational mode, as seen by Frontiera et al. in Fig. 2 of that reference using samples from the same manufacturer, Oxonica. No such dispersive effects are seen in either our pulsed or CW experiments. We will scan over a wider spectral range to confirm that statement, but the data gathered thus far over multiple samples (of S440 and S470 flavors) at different powers (see Fig. $5 \mathrm{~b}$ ) do not show signs of dispersive effects. Frontiera et al. also used a supercontinuum source producing a wide spectral output, unlike our experiments which use (in comparison) a much narrower Ti:Sapphire laser emission. We also use orders of magnitude smaller laser powers (see the discussion at the top of page 3 of our manuscript). These differences may explain the lack of any dispersive response, and the expected reproduction of the spontaneous Raman signal. After a follow up conversation with Dr Van Duyne, we believe the difference may be in the use of a supercontinuum source; further investigation is necessary to pinpoint the cause.

Lauren Jamieson asked: Have you tried these experiments with any other type of nanoparticle in an attempt to improve the detection limit? Alternatively, have you explored any methods to increase the percentage of dimers in the nanoparticle sample, as you say these are the particles contributing to the signal? 
Kevin Hewitt answered: We have used two particle types, S440 and S470, which have almost identical monomer, dimer, and trimer distributions (see Fig. 2). We estimate that tens of particles and picomolar concentrations can be reliably probed. The signals are generated from sample volumes on the order of $10^{4}$ cubic microns (a cylinder of cross sectional area 1 micron $^{2}$ and a path length of $1 \mathrm{~cm}$ ). With a total (monomer, dimer, trimer) concentration of 64 picomolar, and assuming the signal is generated from dimers, trimers and tetramers which make up $10 \%$ of the sample we estimate: $N=6.4 \mathrm{pM} \times$ Avogadro's number $\times 10^{4}$ cubic microns $\approx 10$ nanoparticles. As such the detection limit is quite good.

In conversation with Dr Van Duyne after my presentation, he mentioned that their group has used ultracentrifugation to dramatically increase the dimer/ trimer etc. fraction. We will attempt the same approach.

The Oxonica nanoparticles are the only type we have used for these experiments as the embedded gold nanoparticle is prevented from coming within a diameter of another gold nanoparticle $(60 \mathrm{~nm}$ Au particles are embedded in a silica shell of thickness $30 \mathrm{~nm}$ ), precluding a time dependent change in the enhancement factor; whereas dimers/trimers and tetramers have a fixed relative spacing and a corresponding fixed enhancement factor. The result is a Raman spectrum (from the organic dye coating the AuNP) that is very stable.

On the other hand, bare nanoparticles (e.g. Au/Ag) in solution migrate in a time dependent manner within the radius of an adjacent particle in the focal volume of the laser. The notoriously variable signal in a conventional SERS experiment would result, precluding the likelihood that the small stimulated SERS signal could be extracted. A set of particles fixed on a surface, as in a chain, would not suffer this variability and should exhibit stimulated SERS.
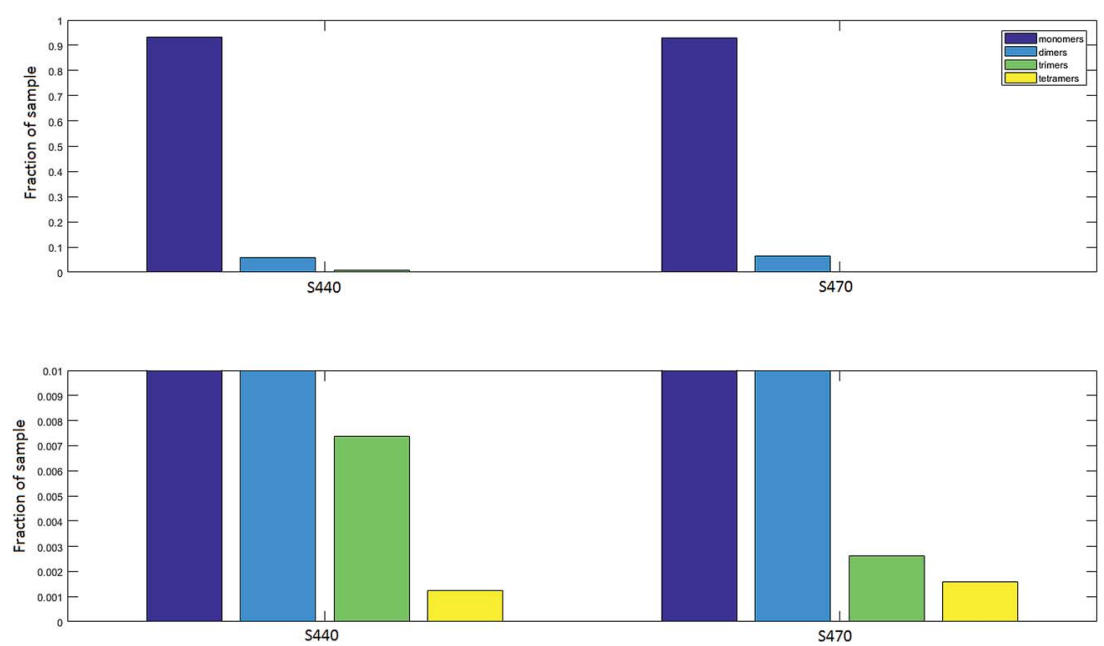

Fig. 2 Histogram showing the fraction monomers, dimers, trimers and tetramers in the S440 and S470 particles of a representative sample (upper panel range 0-100\% and lower panel range $0-10 \%$ to highlight the dimer, trimer, tetramer fractions). 
Richard Van Duyne asked: Why is there no dispersive line shape? The theory from Professor Schatz shows that a dispersive lineshape is an inherent feature of a driven vibrational coherence interacting with a localized surface plasmon. It should be there. The dispersive lineshape is not related to damage, it's still present in the MHz SE-FSRS experiment.

Kevin Hewitt answered: As discussed with you at the conference I think we agreed that while we are using the same Oxonica nanoparticles, there are significant differences in the sources that are used. Reading your paper you point out that if the pulse is on resonance with some other electronic transition one may observe dispersive lineshapes. Indeed your $30 \mathrm{fs}$ probe pulse has a very broad bandwidth (830-1000 $\mathrm{nm}$ ), increasing the likelihood that it would excite other electronic transitions compared with our narrow probe and pump beams. The dimer resonance falls in the range of the bandwidth of your probe, whereas ours does not. I am sending samples to a conference attendee Sylwester Gawinowski who has a femtosecond broadband laser (700-900 nm) which may avoid exciting the dimer resonance because of the smaller long wavelength cutoff. The results of that experiment would help shed light on these differences.

Richard Van Duyne asked: The linewidths look broad. What do you read them as? (And for any other lines, e.g. are the double peaks resolvable?)

Kevin Hewitt answered: The stimulated SERS linewidths are a comparable to the spontaneous linewidths, on the order of $10-15 \mathrm{~cm}^{-1}$ or just over $1 \mathrm{~nm}$. All of the lines we have looked at thus far are not doubled but we plan to present data in an upcoming contribution to examine the resolution issues you rightly point to. Comparing the same sample and identical peaks (Fig. 5a and 6), the pulsed SESRS laser experiments reveal a slightly broader linewidth compared with the $\mathrm{cw}$ se-srs as expected, since the bandwidth of the CW sources are necessarily narrower.

Heike Arnolds remarked: You state in your paper that your signal "exhibits the same characteristics as the spontaneous Raman signal, without a dispersive response". Your signal intensities in Fig. 4 of your paper are around 35 counts per second at maximum. Applying simple Poisson statistics, the error bar for this count rate is $\sqrt{ } 35 \approx 6$ and you show between 8 and 14 points in $5 \mathrm{~cm}^{-1}$ intervals covering a peak which has a FWHM of around $10 \mathrm{~cm}^{-1}$ in spontaneous Raman. While the detection of this signal is a tremendous achievement, I do not think that you have a sufficiently high signal/noise ratio at the moment to make any statement about the lineshape.

Kevin Hewitt answered: I appreciate your question and can provide clarification. The rightmost scale refers to the spontaneous Raman signal (solid line), using a $5 \mathrm{~mW}$ He-He laser, not SRL per the left ordinate. As you can tell from the solid line, the noise is much smaller compared with the signal, and through multiple samples (of S440 and S470 particle flavors) we see the lineshape match that of the spontaneous signal. 
A dispersive lineshape would produce a node when the frequency difference of pump and probe matches the frequency of a molecular vibrational mode, as seen by Frontiera et al. in Fig. 2 of ref. 1 using samples from the same manufacturer, Oxonica. No such dispersive effects are seen in either our pulsed or CW experiments. We will scan over a wider spectral range to confirm that statement but the data gathered thus far over multiple samples (of S440 and S470 flavors) at different powers (see Fig. 2 (below)) do not show signs of dispersive effects. Frontiera et al. also used a supercontinuum source producing a wide spectral output, unlike our experiments which use (in comparison) a much narrower Ti:Sapphire laser emission. We also use orders of magnitude smaller laser powers (see the discussion at the top of page 3 of our manuscript). These differences may explain the lack of any dispersive response, and the expected reproduction of the spontaneous Raman signal.

1 R. R. Frontiera, A.-I. Henry, N. L. Gruenke and R. P. Van Duyne, J. Phys. Chem. Lett., 2011, 10, 1199-1203.

Giuliana Di Martino communicated: Did you try to fix the distance as well? It could be nice to compare the results with Paper $14063^{1}$ and Di Martino et al. ${ }^{2}$

1 G. Di Martino, V. A. Turek, C. Tserkezis, A. Lombardi, A. Kuhn and J. J. Baumberg, Faraday Discuss., 2017, DOI: 10.1039/c7fd00130d.

2 G. Di Martino, V. A. Turek, A. Lombardi, I. Szabó, B. de Nijs, A. Kuhn, E. Rosta and J. J. Baumberg, Nano Lett., 2017, 17, 4840-4845.

Kevin Hewitt communicated in reply: Having read the paper I assume you mean whether we investigated the SRL/SRG as a function of interparticle separation (please see Fig. 3 below). Our measurements were completed on nanoparticles in solution, and the separation between adjacent nanoparticles embedded in the dimers/trimers/tetramers was fixed. It would be of great interest, however, to investigate the stimulated SERS signal with interparticle spacing, utilising the nanoparticle strings described in Herrmann et al. ${ }^{1}$ A detectable signal should be generated, as we estimate a detection limit of tens of nanoparticles (as in our answers to the other questions).

1 L. O. Herrmann, V. K. Valev, C. Tserkezis, J. S. Bernard, S. Kasera, O. A. Scherman, J. Aizpurua and J. Baumberg, Nat. Commun., 2014, 5, 4568.

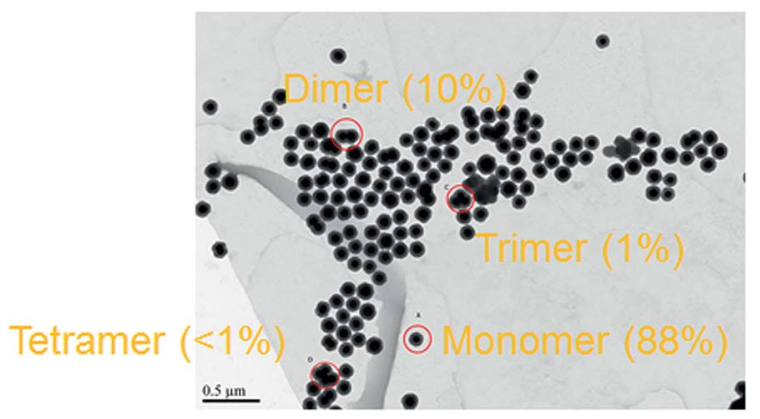

Fig. 3 TEM images of S440 particles demonstrating the relationship between particles in multi-mer particles. 
Ashish Tripathi opened a discussion of the paper by Natalia Martín Sabanés: Regarding Fig. 2 of the article, ${ }^{1}$ the thiophenol SERS spectrum is remarkably different when absorbed from a liquid (water) in comparison to when absorbed from the gaseous (argon) phase. When absorbed from the argon medium, not only is the C-S stretch at $420 \mathrm{~cm}^{-1}$ missing but also the vibrational modes at 1020 and $1080 \mathrm{~cm}^{-1}$ are missing.

Could you comment on why that is?

1 N. Martín Sabanés, A. Elizabeth, J H. K. Pfisterer and K. F. Domke, Faraday Discuss., 2017, DOI: 10.1039/c7fd00164a.

Natalia Martín Sabanés answered: The PhS adsorption is always done in the same way, by immersing the substrate into a PhS-containing solution. Once ready, the sample is mounted into the setup and either purged with argon or immersed in water before measuring. The differences between the argon and water experiments are indeed remarkable. In addition to the mentioned bands missing (or broadened) in the argon experiments, the two bands analyzed shift to higher wavenumbers in water in comparison to the in-argon experiments. The shifts are in the range of $3 \mathrm{~cm}^{-1}$ for the 997 mode and $10 \mathrm{~cm}^{-1}$ for the $245 \mathrm{band}$, as shown in Fig. S2 of the paper's supplementary information. The presence of an aqueous environment can affect the state of the monolayer (and therefore the TER spectra) in different ways. Relative intensity changes might be due to a different adsorption geometry of the molecules at the surface. The band shifts might be produced by a change of the interaction strength between the adsorbates and substrate due to hydration shell formation or chemical reactions such as protonation.

We are currently investigating the origin of these changes, taking in consideration all the bands of the spectra, and DFT calculations of the PhS molecules on $\mathrm{Au}(111)$ in argon and liquid environments. We have not found conclusive evidence to explain the observed changes yet.

Marc Porter said: Your paper indicates that the gold samples were prepared by the method of Clavilier et al. ${ }^{1}$ How large were the (111) single crystal facets of your electrodes? In other words, what is the atomic step density for your surfaces and are the spectra for adsorbates at step sites different from those for adsorbates in the (111) plane?

1 J. Clavilier, R. Faure, G. Guinet and R. Durand, J. Electroanal. Chem. Interfacial Electrochem., 1979, 107, 205-209.

Natalia Martín Sabanés answered: We use high-quality $\mathrm{Au}(111)$ single crystals from Mateck ( $5 \mathrm{~N}$ purity) that are freshly cleaned and flame-annealed before monolayer deposition. Typical terrace widths on our crystals are on the order of $100 \mathrm{~nm}$ with monotonic steps to the next terrace. To ensure minimal adsorption and geometry variation of the target molecules, we select an area in the sample in the middle of a 111 terrace (i.e. an area with the most reproducible adsorbate state) by STM imaging before spectral acquisition.

Marc Porter remarked: Some types of thiolate coatings actually form partial bilayers when prepared from solutions at high reactant concentrations. Have you seen any evidence for such an occurrence in your experiments? 
Natalia Martín Sabanés answered: We do not observe any evidence for multilayer adsorption. After immersing the substrate into the PhS solution, we rinse it carefully to remove multilayers of molecules not chemisorbed to the Au substrate. This procedure for sample preparation is well known and has been reported to work fine for monolayer adsorption. Furthermore, we find no spectral indications of bi- or multilayer adsorption. If a second physisorbed layer of molecules was deposited, it would most likely contaminate the tip during the experiment, especially when ramping at a high current set point and low bias voltage where we expect very small tip-sample distances. Since $\mathrm{PhS}$ chemisorbs very strongly on $\mathrm{Au}$, it would be easy for the second layer of molecules to attach to the gold tip, especially in the case of in-liquid experiments. Tip contamination would result in large SERS contributions to the spectra when the tip is retracted by $20 \mathrm{~nm}$. Since all the tip-retracted spectra, collected repeatedly during experiments at different values of bias and current, are clean with no sign of PhS Raman bands (see retracted spectra in Fig. 2 of the manuscript, in black), we conclude that the tip is not contaminated, pointing towards a sample with a single monolayer of molecules chemisorbed into the gold substrate.

Richard Van Duyne remarked: I have performed similar experiments with similar results. The trends of the tunnelling current and bias agree in ultrahigh vacuum. The difference between argon and water emphasises the advantage of performing TERS in controlled environments. You see the thermal decomposition of thiophenol when it is done in air (the spectral band changes). We had to drop to lower power. It was tricky.

Volker Deckert remarked: A good test for potential multilayer formation (physisorption) would be the appearance of thiol spectra on the tip when the tip is slightly retracted. Probably this has been done?

Natalia Martín Sabanés responded: Indeed, as discussed in the answer to Marc Porter's question, the spectra taken with $20 \mathrm{~nm}$ tip retraction (shown in black in Fig. 2 of the manuscript) show no thiophenol bands. Tip-retracted spectra were taken repeatedly during the course of a bias voltage (or current) ramp indicating that the tip remained clean during the experiments.

Agata Królikowska communicated: When you work with really low absolute values of the bias voltage in a TERS experiment, there is a strong probability that you are really "touching" the molecules with the tip and thus chemical enhancement of TERS signal should not be neglected.

Would you agree that chemical enhancement seems to be underestimated in modeling TERS theory? Maybe the TERS intensity is not only plasmon dependent, and we should take into account that for a close distance between the tip and the sample simply a larger area of the tip is in contact with the molecules, or that when "pressing" the molecules with the tip we can change their polarizability. What is your opinion?

Natalia Martín Sabanés communicated in response: Touching molecules with the tip would pose a high risk of tip contamination. As perviously mentioned in our answers to the preceding questions, we check for tip contamination by 
acquiring retracted spectra that show a clean tip. As the tips are clean of adsorbates, touching the molecules with the STM tip is deemed highly unlikely.

Understanding chemical enhancement is definitely one important open question for the community at the moment and we can only encourage its thorough investigation.

Javier Aizpurua remarked: In the analytical description of the evolution of the TERS intensity as a function of gap separation distance and bias, the model seems to describe the tendencies nicely qualitatively. However, this model relies on several parameters which are very approximate and are left free to fit to the experimental curves. Among all the parameters, the exponent of -10 in the separation distance dependency is left fixed. Why should this be fixed and the other ones left free? Can we obtain any information on the morphology from such a set of free parameters? What should we do for other systems, similar but different?

Natalia Martín Sabanés replied: The model is obtained by combining the tunneling expression with the dependency of the TERS intensities on the gap distance. The dependency of the TERS intensity on the gap distance has been extensively studied by acquiring spectra in tunneling conditions and retracting the tip by accurate distances set by the piezo control (see for example ref. 18 and 20 in our manuscript ${ }^{1,2}$ ). The model with the exponent -10 was found to excellently fit experimental results taken in setups with a similar configuration to ours and was therefore assumed as fixed in our fitting model. It is derived from a simple dipole approximation with a $1 / \mathrm{r}^{3}$ distance dependence, multiplied with the $g^{4}$ TERS enhancement factor, which for a point scatterer results in a $1 / \mathrm{r}^{12}$ distance dependence. Integrating over a finite (2D) nearfield area, we arrive at a $1 / \mathrm{r}^{10}$ distance dependence.

The other parameters in expression (3) of the paper are the tip size - that we can estimate from SEM images of the tips produced in our lab, with a certain error given by the variability in the production process - and the tunneling decay constant and junction resistance at landing position - that are sample dependent, but can be determined experimentally by STM experiments in a simple way and on which a vast amount of literature exists with specific values for a large range of molecules. Thus, since these three parameters have a precise physical meaning, we use the results obtained in free fittings to validate the proposed model. As discussed in the paper, all three parameters are in good agreement with the expected values from literature for all the data series analyzed.

If another system is studied by this approach, the values of the parameters returned by the fittings should be discussed accordingly because different molecule/substrate combinations have different values of the tunneling decay constant and junction resistance, i.e. the values for a similar, but longer, thiol on gold are known to be larger than the ones for PhS. If literature data are not available, one can experimentally determine the tunneling decay constant and junction resistance as described by Toccafondi et al. in ref. 24 of our manuscript. ${ }^{3}$

1 B. Pettinger, K. F. Domke, D. Zhang, R. Schuster and G. Ertl, Phys. Rev. B: Condens. Matter Mater. Phys., 2007, 76, 113409.

2 R. V. Maximiano, R. Beams, L. Novotny, A. Jorio and L. G. Cançado, Phys. Rev. B: Condens. Matter Mater. Phys., 2012, 85, 1-8.

3 C. Toccafondi, G. Picardi and R. Ossikovski, J. Phys. Chem. C, 2016, 120, 18209- 18219. 
Niclas Sven Mueller addressed Natalia Martín Sabanés and Paul Dawson: In the contribution "Novel routes to electromagnetic enhancement and its characterisation in surface- and tip-enhanced Raman scattering" from Paul Dawson in Session $1,{ }^{1}$ it was shown that the plasmon resonance of the tip-sample system strongly shifts when applying a DC bias voltage (see Fig. 7 and 8 of that contribution). As your measurements were performed at a fixed laser excitation wavelength, I would expect that the plasmon resonance wavelength crosses your laser excitation wavelength when increasing the bias voltage. This should lead to a peak in the TERS intensity. However, in Fig. 3 of the paper from Martín Sabanés, ${ }^{2}$ it is shown that the integrated intensity drops monotonously as a function of bias voltage. Could you comment on this? What plasmon wavelength do you expect for your TERS configuration?

1 P. Dawson, D. Frey, V. Kalathingal, R. Mehfuz and J. Mitra, Faraday Discuss., 2017, DOI: 10.1039/c7fd00128b.

2 N. Martín Sabanés, A. Elizabeth, J. H. K. Pfisterer and K. F. Domke, Faraday Discuss., 2017, DOI: $10.1039 / \mathrm{c} 7 \mathrm{fd} 00164 \mathrm{a}$.

Paul Dawson answered: What you say regarding voltage tuning into (and out of) the large resonances in enhancement factor (EF), as shown in Fig. 7 and 8, is essentially correct though the effect cannot be extrapolated directly from these figures. This is because the gap is artificially fixed at $1.0 \mathrm{~nm}$ in the modelling to highlight the influence of the junction field on the Au optical properties in isolation. In practice, if one starts from a certain tunnelling condition and increases the bias under constant current operation, the gap dimension will increase and this will also blue-shift and weaken the gap mode plasmons. So the voltage-dependent response of the EF will represent a convolution of the two effects. This is also discussed in the response to the question in Session 1 from Agata Królikowska.

I'm not quite sure what you mean by the last part of your question. Basically you can use parts (a) of Fig. 7 and 8 to construct EF curves over an arbitrary range of Raman shift for any input wavelength that you choose. For the purposes of meaningful illustration we have chosen some typical laser excitation wavelengths in the red/near-IR.

Natalia Martín Sabanés replied: Our experimental data for both the in-argon and in-water measurements shows a monotonous decrease as a function of increasing $E_{\text {bias }}$. These results are in line with other experimental reports investigating the effect of $E_{\mathrm{bias}}$ on the STM-TERS signal. This behavior suggests that the main parameter affecting the TERS intensity (in the bias region studied between 0.02 and $0.5 \mathrm{~V}$ ) is the gap distance change. Note that in Dawson's paper, larger bias values were showing stronger plasmon shifts.

We expect our gap mode wavelength to be very broad, with resonances at $709 \mathrm{~nm}$ in argon (605 $\mathrm{nm}$ and $905 \mathrm{~nm}$ in water) according to DFT simulations and experimental data published by Bin Ren and coworkers for an essentially identical gap configuration. ${ }^{1}$

1 Z.-C. Zeng, S.-C. Huang, D.-Y. Wu, L.-Y. Meng, M.-H. Li, T.-X. Huang, J.-H. Zhong, X. Wang, Z.-L. Yang and B. Ren, J. Am. Chem. Soc., 2015, 137, 11928-11931.

Jeremy Baumberg opened a general discussion of Volker Deckert's, Kevin Hewitt's, and Natalia Martín Sabanés' papers: I would like to comment that 
models of TERS using a spherical nanoparticle are not believable. A real tip will not radiate due to plasmons travelling up it until all the energy is lost in the metal. I do not understand why this is not discussed more.

A sharp tip is unable to radiate efficiently at all, even though a gap plasmon can form where it is very close to a metal surface. This gap plasmon has a very large wavevector $^{1,2}$ and thus the effective wavelength is $\sim 10 \mathrm{~nm}$. This couples extremely poorly to free space (wavelength $\sim 700 \mathrm{~nm}$ ). Only if there is a neck separating the tip into a nanoparticle and a pillar will there be good radiation. The full discussion and Fig. 7 in "Understanding the plasmonics of nanostructured atomic force microscopy tips" 3 show how this works.

Why is this not more generally known in the TERS community? It suggests other strategies for making good tips.

1 D. O. Sigle, J. Mertens, L. O. Herrmann, R. W. Bowman, S. Ithurria, B. Dubertret, Y. Shi, H.

Y. Yang, C. Tserkezis, J. Aizpurua and J. J. Baumberg, ACS Nano, 2015, 9, 825-830.

2 C. Tserkezis, R. Esteban, D. O. Sigle, J. Mertens, L. O. Herrmann, J. J. Baumberg and J. Aizpurua, Phys. Rev. A, 2015, 92, 053811.

3 A. Sanders, R. W. Bowman, L. Zhang, V. Turek, D. O. Sigle, A. Lombardi, L. Weller and J. J. Baumberg, Appl. Phys. Lett., 2016, 109, 153110.

Paul Dawson answered: I disagree. The tip end does not need to be designed as an independent plasmonic antenna. The point about a gap mode is that there is a strong coupling between the electron gas in the tip and that in the sample - the main dipole moment is directly across the gap and not on the tip or sample independently. There is no requirement that the tip should independently support a localised plasmon mode. Indeed if the tip is designed to support its own mode this will tend to "pull" energy out of the gap which is where you want it. In general, if a metallic tip is brought into nm proximity of a metallic surface gap modes will exist and can be excited under suitable optical or electronic stimulation. In relation to optical excitation these localised plasmon modes of the gap are dipolar in nature and are directly coupled to the free optical field. (The curvature of the tip itself supplies the necessary scale wavevector for coupling.) They may be stimulated by input light, which is exactly what the modelling in Paper 14168 addresses, ${ }^{1}$ and, by reciprocity, will radiate into the far-field. Along with the excitation of the gap modes, surface plasmon polaritons (SPPs) may also be excited which travel along the extended tip profile and the sample surface. These modes do not couple onto the far field but travel away from the junction and are finally absorbed by the metal. To the best of our understanding the gap modes are strongly localised to the junction (over a length scale of a few $\mathrm{nm}$ ) and do not really "see" or couple to the extended tip profile. We discuss these scenarios in some further detail below.

When we say $\mathrm{nm}$ proximity we mean a proximity of $\sim 1 \mathrm{~nm}$, the situation that we address here in STM-TERS. As the tip is withdrawn from the sample the gap modes shift rapidly to shorter wavelengths and the strength of electromagnetic coupling between the tip and sample decreases dramatically. We then arrive at a situation (just a few nm, say $\sim 5 \mathrm{~nm}$ or more separation) where the fields associated with the tip-end probe the (planar) sample - then, what you say about the design of the tip as an independent plasmonic antenna becomes relevant. The mode(s) supported by the tip in free space will no doubt be perturbed - and must be if useful information is to be collected from the sample - but gap modes are not developed. 
Your question also raises points about the propagation of energy away from (or towards) the tip and issues of the sphericity of the tip. In relation to the excitation of gap modes I appreciate that, with good design, it should be more efficient to feed energy into the gap modes by the use of surface plasmon polaritons excited via grating coupling on the sidewall of the tip. Once excited, gap modes may decay by several routes. In addition to the large intrinsic damping in the tip and substrate metal and the desired radiative decay, there is also coupling to SPPs that transport energy away from the gap. The last route divides into two branches, coupling to SPPs that propagate up the body of the tip - in principle this could happen - and to SPPs that propagate away from the tip across the substrate surface. The former may be recovered by a grating etched into the tip sidewall - offering the prospect of effectively building the spectrometer onto the tip with only a lens (for collimation), an edge filter and a CCD camera required in the far field. The latter branch should potentially be of much more concern to the TERS community since SPPs propagating across the surface will sample molecules in their path (a propagation length of $10 \mathrm{~s}$ of $\mu \mathrm{m}$ typically.) If the substrate SPPs scatter to free radiation which is detected by the collection optics then the Raman signal becomes significantly delocalised away from the region of the tip. (We are currently examining this scenario in the context of STM light emission - using a substrate-prism out-coupling geometry - and find that the emission arising from this decay channel is generally quite significant and can, under certain circumstances, exceed that arising from direct emission of the gap modes i.e. the "tip-side" emission.) If the metal surface is very smooth, affording little scattering opportunity to SPPs, and the collection region centred on the tip is small, typically on the order of the diffraction limit, then delocalisation of the Raman signal should not be a great problem. We think these conditions apply to most TERS experiments.

The sphericity or otherwise of nanoparticles and tip-ends was a consistent theme raised throughout the Faraday Discussion by Prof. Baumberg! Given the spatial extent of the gap modes laterally and evanescently into the metal tip and sample we have used a spherical tip as a reasonable first approximation. Our endeavour has been to explain the existence of gap modes in the red and near-IR spectral region. Once a framework is in place to offer such an explanation then the detail of the tip profile will, no doubt, bring about alterations to the gap mode spectral profile and associated field distributions - but this is a secondary issue relative to the very large red-shift that is noted in Paper $14168{ }^{1}$ and has not otherwise been explained to our knowledge. Regarding the field distribution associated with non-spherical structures, a connection has been made between faceted tip profiles and cases of spatial "super-resolution" in some TERS experiments, ${ }^{2-4}$ e.g. by Trautmann et al. ${ }^{5}$ who present the electromagnetic modelling of faceted tip shapes in $\mathrm{nm}$ proximity to a metal surface. The TEM image of such a faceted nanoparticle addressed in that paper doesn't actually depart very significantly from spherical. In any case, the defective region between the crystal facets is modelled as an atomic-scale protrusion at the tip surface that shares the dielectric function of good quality $\mathrm{Au}$ or $\mathrm{Ag}$. The (intra-) molecular resolution that can be achieved in TERS is thus explained in purely optically terms. However, our first inclination would be to look to the feature in the system that is known to be able to give atomic- or molecular-scale resolution, the localised tip sample interaction. In the case of STM-TERS, this is the electron tunnel current filament. Changes in the tunnel current upon encountering a molecule (for various reasons - topographical, local density of states etc. as already outlined in our 
other replies) will drive changes to the gap dimension which in turn modify the localised gap modes and thus the Raman output. The same mechanism-sequence can account for the nm-scale grain boundary resolution in photon maps in STM (see e.g. ref. 6) even though the lateral extent of the gap modes are on the scale of 5$10 \mathrm{~nm}$ or more (with tip radius $20-100 \mathrm{~nm}$ in that work). (Incidentally, we believe that the analysis presented by Trautmann et al. ${ }^{5}$ confuses a "hot" protrusion that happens to be located in a gap with a gap mode plasmon; what is shown in that paper does not have the field structure of a coupled tip-sample gap mode.)

1 P. Dawson, D. Frey, V. Kalathingal, R. Mehfuz and J. Mitra, Faraday Discuss., 2017, DOI: 10.1039/c7fd00128b.

2 J. Steidtner and B. Pettinger, Phys. Rev. Lett., 2008, 100, 236101.

3 R. Zhang, Y. Zhang, Z. C. Dong, S. Jiang, C. Zhang, L. G. Chen, L. Zhang, Y. Liao, J. Aizpurua, Y. Luo, J. L. Yang and J. G. Hou, Nature, 2013, 498, 82-86.

4 J. M. Klingsporn, N. Jiang, E. A. Pozzi, M. D. Sonntag, D. Chulhai, T. Seideman, L. Jensen, M. C. Hersam and R. P. Van Duyne, J. Am. Chem. Soc., 2014, 136, 3881-3887.

5 S. Trautmann, J. Aizpurua, I. Gotz, A. Undisz, J. Dellith, H. Schneidewind, M. Rettenmayr and V. Deckert, Nanoscale, 2017, 9, 391-401.

6 P. Dawson and M. G. Boyle, J. Opt. A: Pure Appl. Opt., 2006, 8, S219-S226.

Volker Deckert replied: I cannot really agree with this statement in general. First of all Taguchi et al. ${ }^{1}$ reported in 2015 that there are issues with too smooth tips and that "rough" tips show a better enhancement. They used a model based on spheres that agrees well with experiments and suggests how to make good tips. It is also clear that the approach to modelling such "real" tips (see Fig. 4 for the tips we generally use and their striking similarity to Taguchi et al.'s proposed shape) by a single spherical particle or by many spheres for that matter is always an approximation. In this context, the proposed "neck" seems to be only another way to approximate the actual tip shape. Admittedly it might explain some properties of "smooth" tips, that are according to Taguchi's conclusions anyway less efficient than rough ones. For our tips (Fig. 4), it seems, however, that a sphere model is closer to reality than a neck. In my opinion the main aspect that has to be considered is the fact that atomic scale roughness (i.e. crystalline facets, edge and corner sites) starts playing a considerable role when such features are closer than $1 \mathrm{~nm}$ with respect to the sample. This was shown for instance by Barbry et al. ${ }^{2}$ using an atomistic description for a $\mathrm{Na}_{380}$ cluster, which is in surprising agreement with our EM based model of a single atom roughness on a sphere ${ }^{3}$ and/or the direct chemical influence of a metal atom on a specific site of a molecule. ${ }^{4}$ It is furthermore important to realise that while a gap mode is usually advantageous, it is by no means necessary to obtain TERS even when no electronic resonances of the molecules are excited (for an almost 10 year old example see ref. 5). The question of why many groups still use smooth massive tips can have several answers. I would speculate that those are easier to implement in STM setups and as long as the enhancement is sufficient this is of course fine.

1 A. Taguchi, J. Yu, P. Verma and S. Kawata, Nanoscale, 2015, 7, 17424-17433.

2 M. Barbry, P. Koval, F. Marchesin, R. Esteban, A.G. Borisov, J. Aizpurua and D. SanchezPortal, Nano Lett., 2015, 15, 3410-3419.

3 S. Trautmann, J. Aizpurua, I. Götz, A. Undisz, J. Dellith, H. Schneidewind, M. Rettenmayr and V. Deckert, Nanoscale, 2017, 9, 391-401.

4 F. Latorre, S. Kupfer, T. Bocklitz, D. Kinzel, S. Trautmann, S. Gräfe and V. Deckert, Nanoscale, 2016, 8, 10229-10239.

5 E. Bailo and V. Deckert, Angew. Chem., Int. Ed., 2008, 47, 1658-1661. 

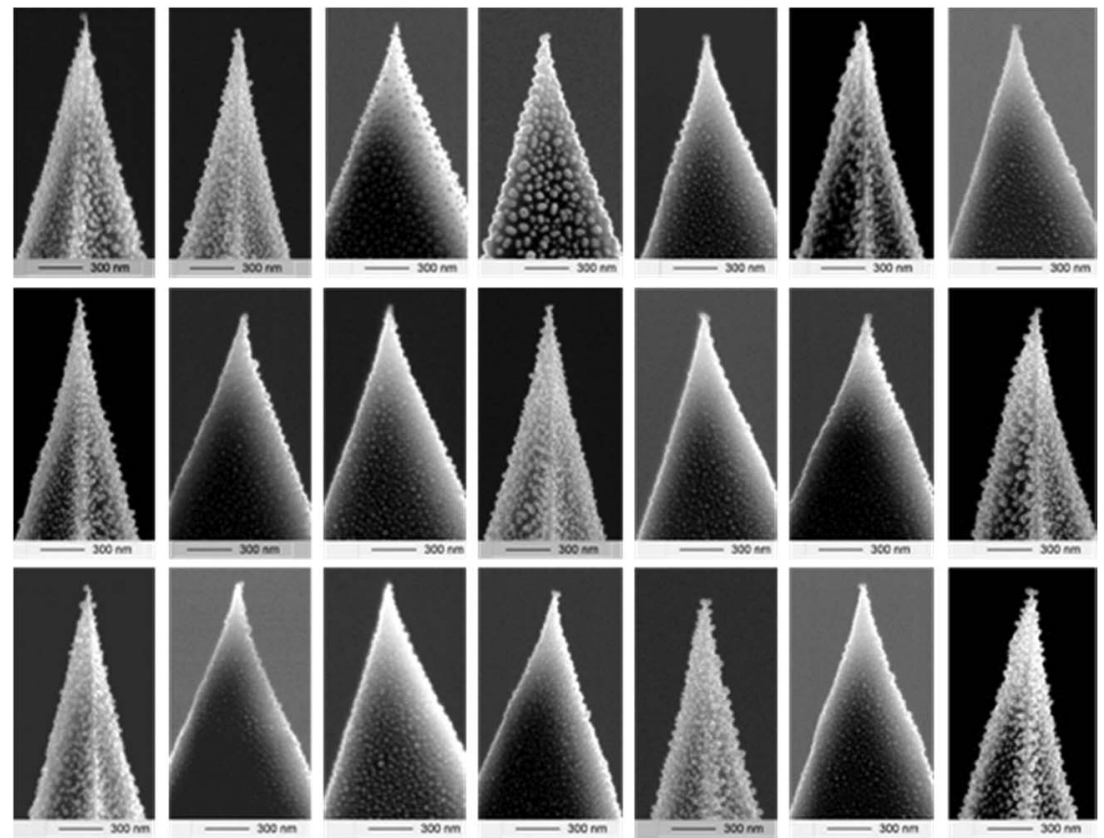

Fig. 4 Typical examples of very efficient TERS tips based on thin silver film evaporation on commercial silicon based AFM probes. The usage of a spherical model for single features seems valid as long as the atomic scale roughness can be neglected.

Richard Van Duyne commented: I agree with Dawson. The gap mode is key to making TERS work; but, that makes it difficult to measure the plasmon resonance. If you do TERS excitation spectroscopy, the gap mode plasmon has the same basic structure as two spheres interacting. The gap mode exists for other materials, so you can predict for different tip-substrate combinations, for example a gold tip and silver substrate.

Jeremy Baumberg answered: Unfortunately, I do not believe that the gap modes calculated for TERS using two spheres are correct. This is because the difference between a tip and a sphere is that to get good plasmon confinement in the gap you need the charges on the other side of the sphere, and these are shorted out by the connecting tip. Only if the tip has particular shapes with necks can this be improved, and is perhaps the reason why grains at the end of tip structures seem to work better. This can be seen in both experiment and models in our recent paper, "Understanding the plasmonics of nanostructured atomic force microscopy tips". 1

1 A. Sanders, R. W. Bowman, L. Zhang, V. Turek, D. O. Sigle, A. Lombardi, L. Weller and J. J. Baumberg, Appl. Phys. Lett., 2016, 109, 153110.

Christian Kuttner addressed Richard Van Duyne: You commented that silver is the best base material for TERS, independent of the tip material. Is this related to the low damping of Ag? 
Richard Van Duyne responded: Yes - Ag has the least damping of any plasmonic material.

Zhong-Qun Tian addressed Jeremy Baumberg and Richard Van Duyne: How do we fully correlate the simulation model to the real system, because some simplified models may not well represent the experimental systems? This becomes more important for quantitative studies of the enhancement factor, which is determined by several factors. My colleague, Dr S. Y. Ding has found that for tip enhancement studies, if the simulation considers the whole metallic tip as a nanoparticle, the enhancement factor is much stronger than that from the experimental observations. I wonder how to design and optimize the tip structure for the best enhancement that is guided by simulation.

Jeremy Baumberg replied: Doing proper simulations is crucial for understanding TERS. As I noted, it is not correct to use a spherical particle or ellipsoidal particle as the tip - it is completely wrong for two reasons: the top of the particle exhibits surface charge, allowing light to couple in and out to the tip efficiently; it also changes the surface plasmon resonance spectral positions erroneously. The presence of the bulk of the metal tip stops light coupling in and out, and all the plasmons travel along the tip surfaces being attenuated and lost as heat.

It is important thus to use a considerable part ( $>10$ microns) of the tip structure in the simulations. If you look at our recent paper "Understanding the plasmonics of nanostructured atomic force microscopy tips", ${ }^{1}$ you will see that the optimum tip has a neck of at least half the diameter. In this case the outcoupling is strong and the spectral resonances are well established. In my view it is likely that this is where the irreproducibility of TERS comes from, and Ag grains at the end of the tip sometimes form this structure.

1 A. Sanders, R. W. Bowman, L. Zhang, V. Turek, D. O. Sigle, A. Lombardi, L. Weller and J. J. Baumberg, Appl. Phys. Lett., 2016, 109, 153110.

Jean-Francois Masson asked: To further contribute to the discussion about the plasmon on the tip of a TERS probe, we have recently measured white light reflection on the tip and on the springboard of a gold-coated AFM cantilever (Fig. 5). The excitation was performed from the silicon nitride side of the tip. We observed what looks like a strong plasmon resonance only at the tip, from the absorbance at $642 \mathrm{~nm}$ only seen at the tip and not on the springboard. The plasmon resonance changed between air (642 $\mathrm{nm}$ ) and water ( $648 \mathrm{~nm}$ ), providing further support for the excitation of a plasmon resonance is at the tip in these conditions. This data could provide more insight to the discussion about the plasmon resonance at the tip of a TERS probe.

John Lombardi responded: This is an interesting observation. Do you suggest that the SiN portion of the tip is supporting a plasmon resonance? Do you see the phonon modes of SiN in the Raman spectrum?

Jean-Francois Masson answered: Indeed. These tips were gold coated with a thickness on the order of $50 \mathrm{~nm}$, such that we propose they act as a micrometer-size surface plasmon resonance sensor. The illumination is from the SiN tip and we believe the excitation of the plasmon is analogous to the Kretschmann configuration of SPR. 
We have not measured the Raman spectrum at these tips, but we did not observe phonon modes on other tips.

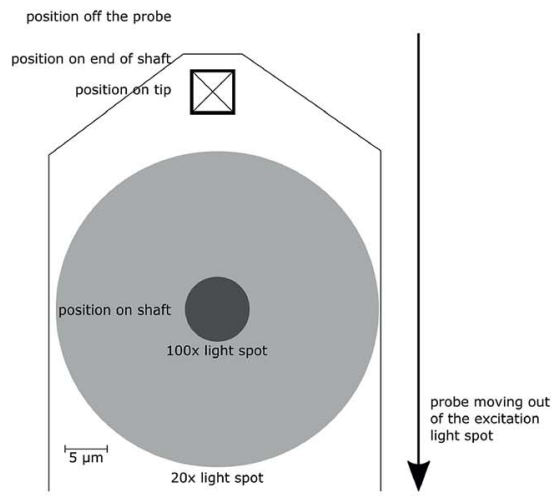

(a)

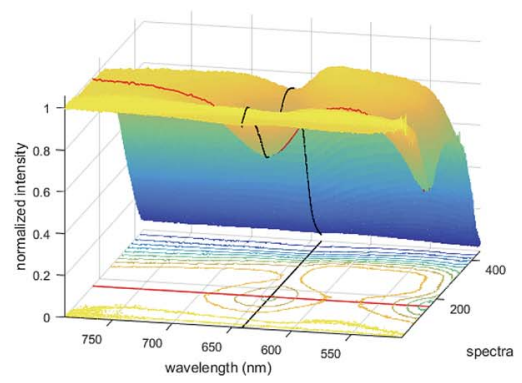

(c)

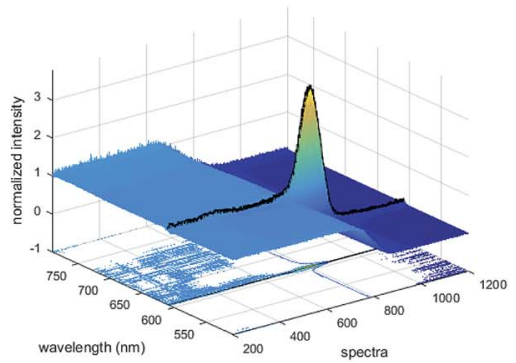

(b)

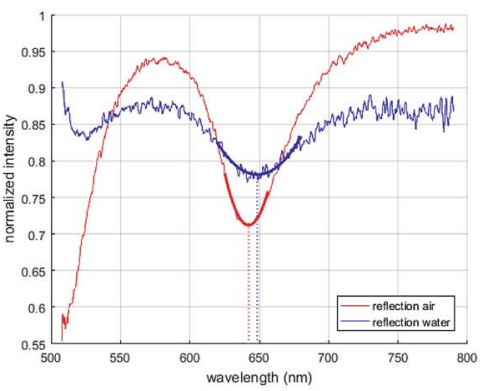

(d)

Fig. 5 (a) Sketch of a probe with shaft and tip and the excitation light spots using different objectives. The light spots are fixed in place and the movement of the probe during a measurement is indicated by the downward facing arrow. In the illustrated situation the shaft of the probe would be measured. (b) Normalized reflection spectra of white light from an uncoated $\mathrm{Si}_{3} \mathrm{~N}_{4}$ AFM probe. The black line indicates wavelength position $608 \mathrm{~nm}$. (c) Normalized reflection spectra of white light from a LSPR-AFM probe with a gold coating of $45 \mathrm{~nm}$. With an increasing number of spectra the probe is moving through and out of the excitation light. The position of the plasmon dip $(641 \mathrm{~nm})$ is highlighted with black and red lines. (d) Comparison of plasmon dip in air and water. Exact wavelengths of highlighted positions in increasing order: $642 \mathrm{~nm}$ and $648 \mathrm{~nm}$.

Christian Kuttner communicated a query regarding the paper by Volker Deckert: The photocatalysis of plasmon-induced chemical reactions in TERS relies on the hot spot intensity, which depends on the sharpness of the tip. Could you comment about the importance of the tip sharpness and geometry?

Volker Deckert communicated in reply: Please see my answer to the earlier question on tip shapes by Jeremy Baumberg.

Niclas Sven Mueller opened a discussion of the paper by Kallie Willets: In the first part of your paper where you consider a system free of surface roughness, you explain the asymmetry of the emission pattern with the additional excitation of an in-plane plasmon mode. I would expect the in-plane dipole in the nanosphere to 
induce an image dipole in the substrate pointing in the opposite direction, which gives rise to a dark plasmon mode. As dark plasmon modes cannot radiate into the far field, I would expect that there is no influence of in-plane plasmon modes on the emission pattern?

Katherine (Kallie) Willets answered: The argument stems from the presence of the molecule in the gap, which breaks the symmetry of the system and stops it behaving like an ideal gap mode substrate and allows formerly dark modes to become bright (a discussion on this point can be found in ref. 1). This is critical, especially in the single molecule regime, where the extent of the symmetry breaking is much more exaggerated than in the high coverage regime, where these effects are averaged out and the system tends to appear more "wellbehaved" (e.g. consistent with predictions using plane wave excitation).

1 S. L. Kleinman, B. Sharma, M. G. Blaber, A.-I. Henry, N. Valley, R. G. Freeman, M. J. Natan, G. C. Schatz and R. P. Van Duyne, J. Am. Chem. Soc., 2013, 135, 301-308.

Giuliana Di Martino asked: You should also expect donut shapes in the dark field images. Why are these not observed?

Katherine (Kallie) Willets answered: To obtain the dark field images, we are using a high numerical aperture dark field condenser, but a low numerical aperture collection lens $(\mathrm{NA}=0.6)$. To create the donut shape, high angle light must be collected. Thus we do not expect to see donut shapes in the dark field scattering due to the optics of the system.

Bart de Nijs said: Comparing in absolute intensities, is the surface roughness detrimental or beneficial to the observed signal strength? Is there any correlation between the surface roughness and features in the dark-field scattering spectra?

Katherine (Kallie) Willets responded: We have not taken dark field scattering spectra, so cannot comment on the relationship between spectral features and surface roughness, although the inverse system ("rough" or faceted nanoparticles on a smooth film) does show spectral features that could serve as diagnostic peaks (see ref. 1). At present, we do not have enough statistics to verify whether surface roughness is helpful or not.

1 M.-E. Kleemann, J. Mertens, X. Zheng, S. Cormier, V. Turek, F. Benz, R. Chikkaraddy, W. Deacon, A. Lombardi, V. V. Moshchalkov, G. A. E. Vandenbosch and J. J. Baumberg, ACS Nano, 2017, 11, 850-855.

\section{Sebastian Heeg commented:}

1. Why do you use circular polarization to couple to the out-of-plane gap plasmon? Radial polarization has an out-of-plane component at the center of the beam which should facilitate coupling to the gap mode.

2. Have you tried to use template-stripped gold surfaces to verify your hypothesis? I think they are available in roughnesses with an RMS of around $0.5 \mathrm{~nm}$.

Katherine (Kallie) Willets responded:

1. Our current experimental setup does not have the correct optics to generate radially-polarized light. Although our current excitation geometry is inefficient, we have sufficient $z$-polarization due to the high numerical aperture objective we use to 
provide non-zero out-of-plane excitation. Introducing radially-polarized excitation would increase the signal-to-noise and improve the accuracy of our fits.

2. We have not tried template-stripped gold, although the silver films we prepared with an RMS roughness of $0.3 \mathrm{~nm}$ have similar characteristics to gold films prepared in this manner.

Mike Hardy commented: Hi Kallie, in this investigation Nile Blue is used; have you attempted these experiments with other molecules? With a larger molecule one would suppose that the out-of-plane angle, $\theta$, would converge beyond the 2030 degree range observed in this study (assuming the surface roughness could be reproduced at 1.3-2.2 nm RMS). This might be a nice confirmation of the proposition as depicted by Fig. 9 in your paper, ${ }^{1}$ whereby the increased asymmetry in $\theta$ in the data in Fig. 1 and 6 of the paper (in comparison with a previous data set) is attributed to an increase in surface roughness. Is this correct? Many thanks.

1 P. B. Joshi, T. P. Anthony, A. J. Wilson and K. A. Willets, Faraday Discuss., 2017, DOI: 10.1039/c7fd00163k.

Katherine (Kallie) Willets answered: So far we have not attempted this experiment with other molecules, although it would be simple to implement other probes or even molecular spacer layers. Based on the discussion and the many questions, the conclusion is that surface roughness is but one variable that can impact how the light is outcoupled by this system. In our case, we saw a correlation between the two, but like much of SERS, there are other variables that will also contribute, including the molecular orientation, position, surface facets of the nanoparticles, etc.

Jeremy Baumberg asked: I wanted to raise that there is another very significant factor in this nanoparticle-on-mirror (NPOM) geometry, that affects the emission pattern you see.

If you look at "Revealing Nanostructures through Plasmon Polarimetry", 1 you will see that the NPoM facet anisotropy plays a large role. Particles are not spherical and looking at both the in-plane and vertical parts of the dipole emission shows clearly how the bottom facet can determine the theta and phi you measure (through the splitting of the in-plane modes). (Note that in the paper it is both incoupling and outcoupling, whereas for you it is outcoupling, but it has the same effects). Is there a way to distinguish between this facet effect and the roughness effect you mention?

1 M.-E. Kleemann, J. Mertens, X. Zheng, S. Cormier, V. Turek, F. Benz, R. Chikkaraddy, W. Deacon, A. Lombardi, V. V. Moshchalkov, G. A. E. Vandenbosch and J. J. Baumberg, ACS Nano, 2017, 11, 850-855.

Katherine (Kallie) Willets responded: This is an excellent question, and one that requires further data acquisition and analysis to fully resolve. That said, however, our results do suggest that the surface roughness of the underlying thin film (e.g. the mirror) plays a role in determining the symmetry of the out-coupled light. This conclusion comes from the data comparing the out-of-plane angle, theta, for silver films with an RMS roughnesses of $\sim 2 \mathrm{~nm} v s .0 .3 \mathrm{~nm}$ in which we saw theta values of $\sim 25$ degrees $v s$. $<10$ degrees.

Jeremy Baumberg said: As you bleach out emitters in the low concentration regime, do the results change for theta/phi? It might be that you see emitters from 
different regions in the gap. Or it might be that each structure completely fixes the asymmetry.

Katherine (Kallie) Willets answered: We do see some relationship between intensity fluctuations that could be signatures of different molecules turning on and off, and changes in the values of theta and phi, but surprisingly, the two are not completely correlated (see Fig. 6 and 7 where different trends are indicated). So we believe that there are multiple effects that contribute to the observed fluctuations in these values, ranging from the position of the molecule(s) within the gap (especially as that position changes relative to any surface defect sites) to the orientation of the molecule(s). Unfortunately, we don't see distinct photobleaching events that allow us to distinguish one molecule from another. Isotope-edited probes and correlated SERS spectra would help address this issue.

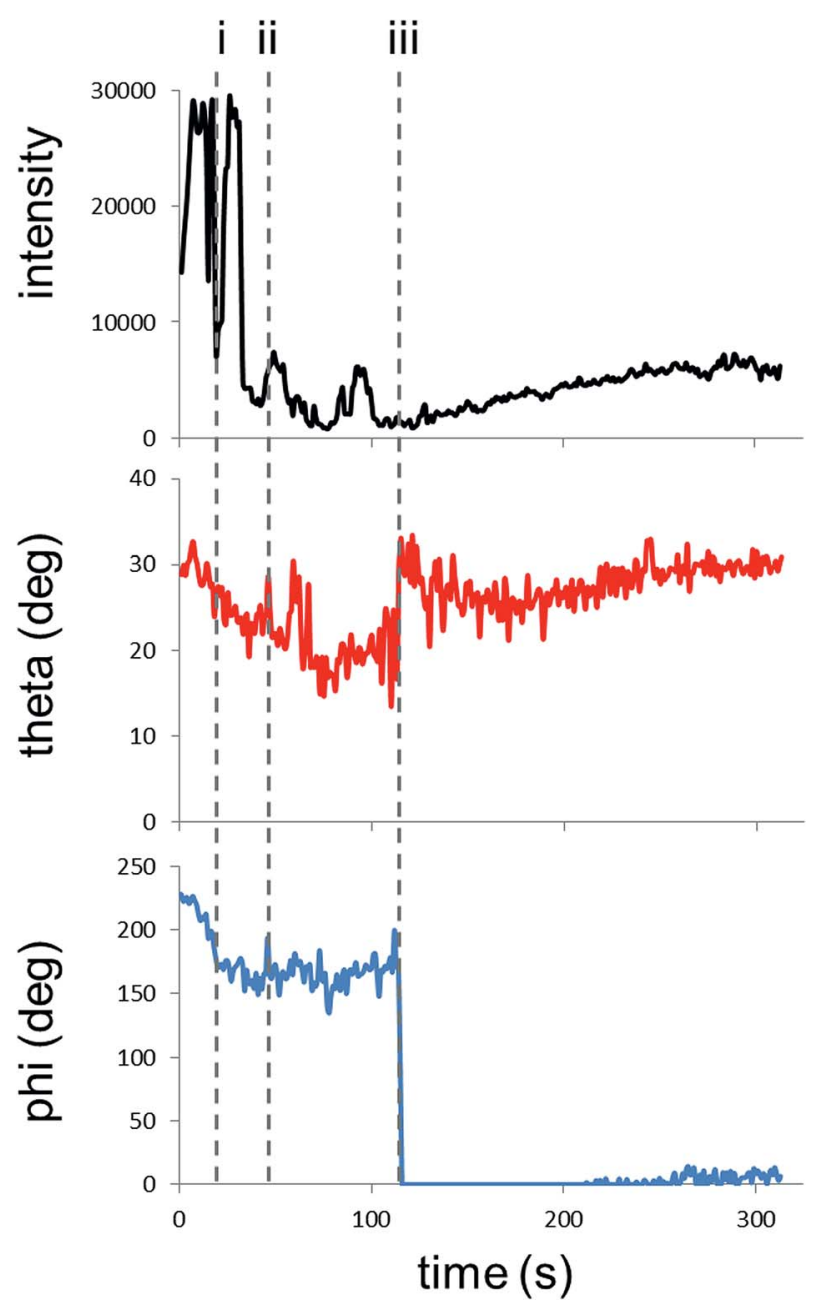

Fig. 6200 nM. NP 1 in manuscript. Event i. Drop in intensity shows no change in $\theta$, but a change in $\varphi$. Event ii. Rise in intensity shows a (transient) increase in both $\theta$ and $\varphi$. Event iii. No change in intensity, but a jump in $\theta$. $\varphi$ converges to zero (lower bound) until $\sim 220$ seconds when values fluctuating around zero are recorded. 
Rohit Chikkaraddy asked: In the SERS images shown in Fig. 1, 8 and 10 of the paper, how much is the contribution from the fluorescence of the molecule or inelastic scattering from the plasmonic metal and how much is the Raman signal? How do you deconvolute these?

Katherine (Kallie) Willets responded: In the current implementation, we cannot deconvolve any contributions from the plasmonic metal from the SERS signals. However, Fig. 1 parts E and F show the SERS and dark field images, respectively, from a sample in which a low concentration of Nile Blue is introduced. Although there are many nanoparticles present on the surface, as confirmed by the dark field image, they generate a negligible signal in the correlated SERS image. If we had substantial contributions from the metal, we would expect all of the nanoparticles in the dark field image to be visible under laser illumination. Thus, we expect the contribution from the metal to be fairly

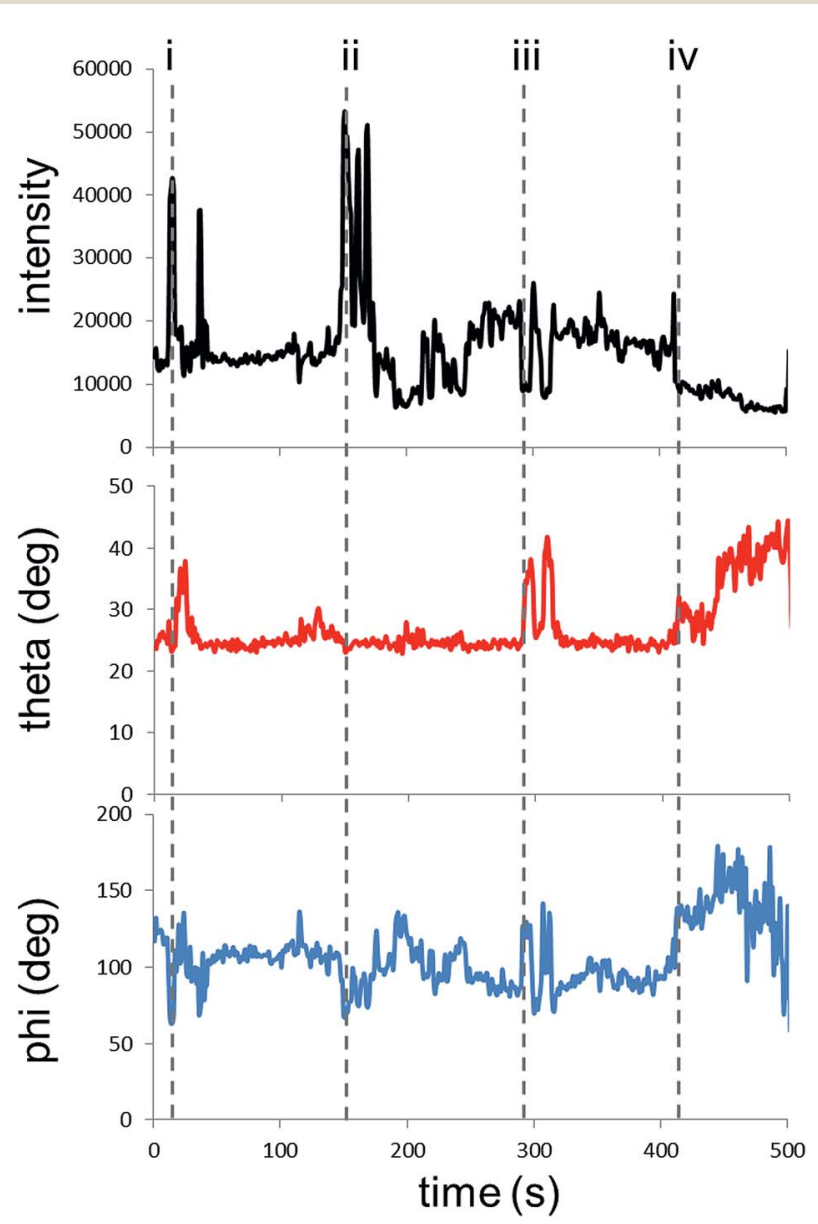

Fig. 7200 nM. NP 2 in manuscript. Event i. Jump in intensity accompanied by higher value of $\theta$ and lower value of $\varphi$. Event ii. Jump in intensity has no associated change in $\theta$ but a large drop in $\varphi$. Event iii. Drop in intensity accompanied by higher value of $\theta$ and higher value of $\varphi$. Event iv. Drop in intensity accompanied by higher value of $\theta$ and higher value of $\varphi$. 
weak, and the signals that we observe are dominated by SERS. We could confirm this by performing correlated spectral measurements. We also note that $643 \mathrm{~nm}$ illumination was used for these experiments, which does not generate a substantial background from silver, in comparison to blue/green excitation where backgrounds can be quite high (see, for example, our previous work ${ }^{1}$ where we relied upon fluctuations in the SERS signals to allow us to deconvolve the silver background from the SERS signals).

1 M. L. Weber, J. P. Litz, D. J. Masiello and K. A. Willets, ACS Nano, 2012, 6, 1839-1848.

Rohit Chikkaraddy said: What are the wavelengths of the molecular absorption and emission with respect to the coupled plasmon resonance?

Katherine (Kallie) Willets answered: While we did not measure the plasmon resonance of the substrates, we expect them to be in the near IR ( $>700 \mathrm{~nm}$ ) which is spectrally inaccessible with our current dark field setup (although it could be modified to access this spectral range). As such, the particles appeared blue by eye, consistent with scattering dominated by quadrapole or transverse scattering (see ref. 1). The absorption and emission of Nile Blue dye is environmentally dependent, but in water it has an absorption maximum near $635 \mathrm{~nm}$ and emission maximum near $670 \mathrm{~nm}$. We are exciting on this molecular resonance and find that the SERS is $\sim 10 \times$ brighter when excited at $643 \mathrm{~nm}$, compared to 532 and $488 \mathrm{~nm}$. Thus, we expect both resonance enhancement as well as enhancement due to coupling to the gap mode.

1 M.-E. Kleemann, J. Mertens, X. Zheng, S. Cormier, V. Turek, F. Benz, R. Chikkaraddy, W. Deacon, A. Lombardi, V. V. Moshchalkov, G. A. E. Vandenbosch and J. J. Baumberg, ACS Nano, 2017, 11, 850-855.

Christian Kuttner said: The emission patterns presented for out-of-plane polarized gap modes look like a series of concentric, alternating bright and dark rings. A similar pattern can be found for Newtonian rings. A phenomenon in which, depending on the refractive indices, an interference pattern is created by the reflection of light between two surfaces - a spherical surface and an adjacent touching flat surface. Can you rule out that changes in the refractive indices might contribute to your emission pattern?

Katherine (Kallie) Willets replied: To see these types of interference effects, a single wavelength excitation source would be necessary to get clear rings; in the case of multiple wavelengths, you would expect the rings to be smeared out due to the wavelength dependence of the interference. In our study, we are imaging SERS emission and thus the images are created over a range of wavelengths, making interference an unlikely effect. In addition, our results match extremely well with previously observed gap mode substrates (see ref. 41 and 42 in the manuscript $t^{1,2}$ ) as well as predictions for a primarily $z$-oriented emitter.

1 S.-Y. Chen, J. J. Mock, R. T. Hill, A. Chilkoti, D. R. Smith and A. A. Lazarides, ACS Nano, 2010, 4, 6535-6546.

2 L. Du, D. Tang, G. Yuan, S. Wei and X. Yuan, Appl. Phys. Lett., 2013, 102, 081117. 
Zhong-Qun Tian asked: Your approach is very interesting and important. I wonder if you could change your tip with a different metallic alloy and/or modify the tip surface with different metallic atoms/clusters. Based on our simulations, nanocubes can create a better hot spot so the enhancement of the Raman intensity is much higher than with nanospheres. Can you change the plasmonic nanostructured tip to a nanocube?

Katherine (Kallie) Willets replied: This approach is completely universal for any substrate with emission coupled to a gap plasmon mode. We are starting to explore some of these different nanoparticle geometries to see what types of effects the shape has on the outcoupled SERS emission pattern, beginning with cubes following your suggestion as well as after further discussion with Jeremy Baumberg.

Jean-Francois Masson said: Do you have a molecular spacer to ensure a gap between the nanoparticle and the surface? Is it possible that the gap mode would not be available due to a lack of space or the nanoparticle touching the surface?

Katherine (Kallie) Willets answered: The only spacer that is used for these samples is the SERS reporter itself. Thus, especially for the lower concentration samples, there is a strong possibility that the nanoparticle is in direct contact with the underlying silver film. This would create a crevice in which the molecule(s) could reside. This crevice would still enable single molecule SERS (see, for example, R. P. Van Duyne and coworkers ${ }^{1}$ ), but would also have a strong out-ofplane polarization component which would generate the donut-shaped emission pattern. In fact, it is by virtue of the observation of the donut shape that we can definitively state that we have an out-of-plane dipole component associated with the SERS emission (see, for example, ref. 2). So regardless of whether this is a true "gap mode" or perhaps a "crevice mode", we are able to confirm a strong out-of-plane component to the emission.

1 S. L. Kleinman, E. Ringe, N. Valley, K. L. Wustholz, E. Phillips, K. A. Scheidt, G. C. Schatz and R. P. Van Duyne, J. Am. Chem. Soc., 2011, 133, 4115-4122.

2 E. H. Hellen and D. Axelrod, J. Opt. Soc. Am. B, 1987, 4, 337-350.

Christian Heck asked: In your answer to a previous question you discussed how the gap distance between the particle and surface is determined by the dye molecules. To follow up on that: Could it be that for some structures the particle is in direct contact with the surface and that the site of strongest enhancement would thus be located at the respective crevice? How would this influence the asymmetry of the doughnut-shaped emission patterns?

Katherine (Kallie) Willets responded: Please see my previous reply for a discussion of this "crevice mode". As an additional point, I would expect the crevice geometry to induce asymmetry in the system, similar to the cartoon schematic in Fig. 9A of the manuscript.

Bart de Nijs said: Using a $640 \mathrm{~nm}$ laser, could it be possible you are exciting a higher order mode preventing you from probing the centre of the plasmonic hot-spot/cavity? 
Katherine (Kallie) Willets replied: Even if we are exciting a higher order mode, one would expect it to appear symmetric, unless there was some structural/ molecular feature to induce the asymmetry. While this is difficult to probe optically, a lovely paper from Garcia de Abajo using cathodoluminescence illustrates this. ${ }^{1}$ Moreover, our technique does not probe what is being excited, but rather what is being emitted. So the SERS image reflects how light is being directed into the far field by the gap mode substrate. In this case, the (a)symmetric donut shape is consistent with an oriented dipole at an interface (see Fig. 5 of the manuscript), leading us to conclude that the emission is dominated by a radiating dipole plasmon mode.

1 N. Yamamoto, S. Ohtani and F. J. G. de Abajo, Nano Lett., 2011, 11, 91-95.

George Schatz said: Regarding the sin(theta) weighing of the distribution measured, do you have the data to see this aspect (small theta) of the distribution?

Katherine (Kallie) Willets answered: Fig. 8 contains two plots showing the relationship between intensity and $\cos ^{2}(\theta)$. We chose to plot this relationship

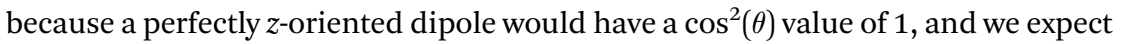
the intensity to track with this value squared for a dipole emitter. In one example shown, the highest intensity SERS events are all associated with large values of $\cos ^{2}(\theta)$, while in the other case, high intensity events occur at multiple values of $\cos ^{2}(\theta)$. Thus, other effects such as the molecule orientation, molecule position relative to any surface defects, particle faceting, etc. may be generating additional effects that this simple relationship cannot account for.
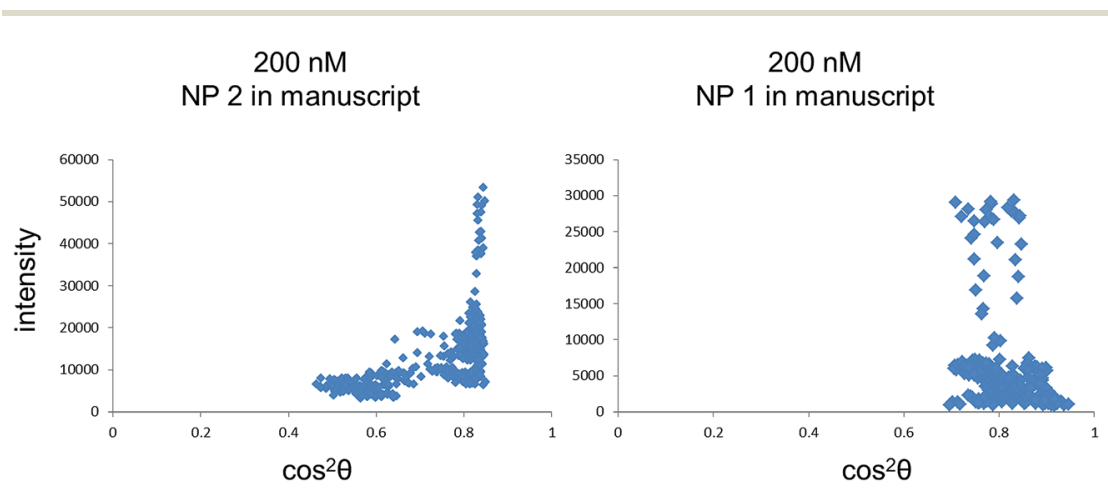

Fig. 8 Plots of SERS intensity vs. $\cos 2 \theta$ for the nanoparticles from the manuscript as indicated.

Sylwester Gawinkowski communicated: Could you comment on the high distribution of intensities in the dark field images (Fig. 1 in the paper)? For very similar nanoparticles deposited on the surface, one can expect similar intensities of dark field scattering images.

Katherine (Kallie) Willets communicated in reply: The higher intensity regions are associated with particle aggregates. We did not include any of these aggregates in our data analysis. 
Joshua Edel opened a discussion of the paper by Kei Murakoshi: What potentials can you go up to, and what is the electrochemical window? Furthermore, do you have issues with the stability of ITO, and if so how do you deal with this?

Kei Murakoshi responded: A more negative polarization than $-1.0 \mathrm{~V}$ results in damage to the ITO electrode. We carefully examined the potential region where the ITO and metal nanostructure were not damaged by electrochemical reactions, such as ITO reduction and hydrogen evolution.

Niclas Sven Mueller asked: In a recent paper by Eric $\mathrm{Le} \mathrm{Ru}^{1}$ it was shown that the molecular absorption typically shifts when the molecules adsorb on a metal surface. How can you be sure that the extinction spectrum of the Au-NSL substrate with molecules (Fig. 2 of your manuscript, ${ }^{2}$ top) is not just the sum of the extinction of the Au-NSL substrate without coverage (Fig. 2, middle) and the shifted absorbance of the molecules (Fig. 2 bottom - blue shifted)?

1 B. L. Darby, B. Auguié, M. Meyer, A. E. Pantoja and E. C. Le Ru, Nat. Photonics, 2016, 10, 40-45.

2 H. Minamimoto, F. Kato, F. Nagasawa, M. Takase and K. Murakoshi, Faraday Discuss., 2017, DOI: 10.1039/c7fd00126f.

Kei Murakoshi responded: We should consider the effect of the change in the absorption spectrum of the dye on the metal nanostructure as you suggested. The present system shows an energy shift downward both in the upper and lower branches of the splitting as in a previously reported anti-crossing plot of the dye exciton energy vs. LSP energy (Fig. 3 in ref. 1). The shift may involve the effect of the change in the dye absorption. Even in that case, electrochemical control of the coupling is confirmed by the change in the splitting energy as the function of the electrode potential.

1 F. Nagasawa, M. Takase and K. Murakoshi, J. Phys. Chem. Lett., 2014, 5, 14-19.

Niclas Sven Mueller asked: What is the impact of the electrode potential on the intrinsic resonance of the molecule itself? Could this have shifted the absorbance of the molecules in the measurements that gave rise to Fig. 4 in your manuscript?

Kei Murakoshi responded: At the present, we assume that the energy of the resonance of the molecule is not affected by the electrode potential. The observed change in the splitting is quantitatively explained by the electrochemical potential-dependent changes in the number of molecules and the LSP energy of the metal nanostructure covered by dye in an electrolyte solution.

Marc Porter asked: Where is the potential of zero charge for your system? This question is actually aimed at understanding if the adsorbates are stable under your test conditions.

Kei Murakoshi responded: The potential of zero charge for the Au electrode is more positive than $0 \mathrm{~V} v s$. $\mathrm{Ag} / \mathrm{AgCl}$. A cationic dye before reduction could be adsorbed on the metal surface under the present conditions at a relatively negative potential (from $0 \mathrm{~V}$ to $-0.8 \mathrm{~V}$ ). To improve the stability of the dye 
adsorption, we should introduce surface modification of the metal nanostructure using the self-assembly method etc.

Alastair Wark asked: Is the switch between the oxidised and reduced forms of HITC reversible?

Kei Murakoshi replied: At this moment, the splitting is not completely recovered at re-oxidation after negative polarization. This could be due to the desorption or the electrochemical decomposition of reduced dye radicals on the metal surface in an aqueous solution. We expect that a system using non-aqueous solutions may improve the reversibility.

Jeremy Baumberg said: You show a very peculiar tuning curve. Since you believe that all the molecules are bleaching/reducing at a more negative potential $(-0.8 \mathrm{~V})$ would you not expect the splitting in the spectra to completely collapse/go away? This does not seem to happen. Can you plot the SERS strength (with backgrounds removed) $v s$. the square of the estimated splitting, which should be a straight line though the origin, but does not look like it will be.

Kei Murakoshi answered: In the present system, LSP could be also coupled with excitons of dye molecules not attached the surface of the metal nanostructures, as well as those contacted with the surface. The electrochemical potential may control the redox states of only the dyes adsorbed on the surface. Thus, it could be possible that the splitting is retained at a more negative potential that the redox potential of the dyes. Quantitative analysis of the correlation between the Raman intensity and the change in the splitting energy may provide information on the spatial distribution of dye molecules in the LSP field.

Javier Aizpurua remarked: There is often a misconception in electrochemistry about the effect of charging particles in the shifts of their optical spectra. One might think that if you negatively charge a particle, the charge density is increased homogeneously, and then the plasma frequency of the metal increases, and therefore we have a blue-shift of the surface plasmon response of the particle due to this effect. This is wrong. When one performs full quantum calculations, based on Time-Dependent Density Functional Theory (TDDFT), of metallic particles in a vacuum (we did this with Andrei Borisov in a recent Langmuir paper ${ }^{1}$ ) one can see that the excess of charge, and therefore the electron density, is not spread homogeneously over the whole particle (see Fig. 9). This excess of charge is on the interface of the cluster/gap/etc. and is not held distributed inside the bulk. Therefore, the energy does not change at all in such a charging process, and neither does the spectrum (see Fig. 9). These are considerations in a vacuum. In electrochemical environments, one has the electrolytes and dipolar layers, therefore the experimentally measured shifts must necessarily be associated with effects of the chemical (dielectric) change of the environment.

You show considerations on plasmon shifts to trace strong coupling as you apply an increasing voltage. Along the lines of what I commented above, do your results consider the source of the plasmonic shifts? Do you know why and how the surface plasmon changes? Do you see differences in using different metals? In Fig. 9 I show how $\mathrm{Na}$ or $\mathrm{Ag}$ particles behave totally differently, for example. My point is about being careful when detecting and interpreting plasmonic shifts in electrochemistry, 
which might be due to other effects. Still, of course you can have strong coupling, as you show in your paper, if you manage to engineer your plasmon as a function of voltage, but do we really know why and how it shifts the way it does? Also, why in your results do the experimental points bend and shift inside each brand like they do, instead of following the line of the theoretical prediction?

1 M. Zapata Herrera, J. Aizpurua, A. K. Kazansky and A G. Borisov, Langmuir, 2016, 32, 28292840.

\section{Electron charge density in metallic clusters}

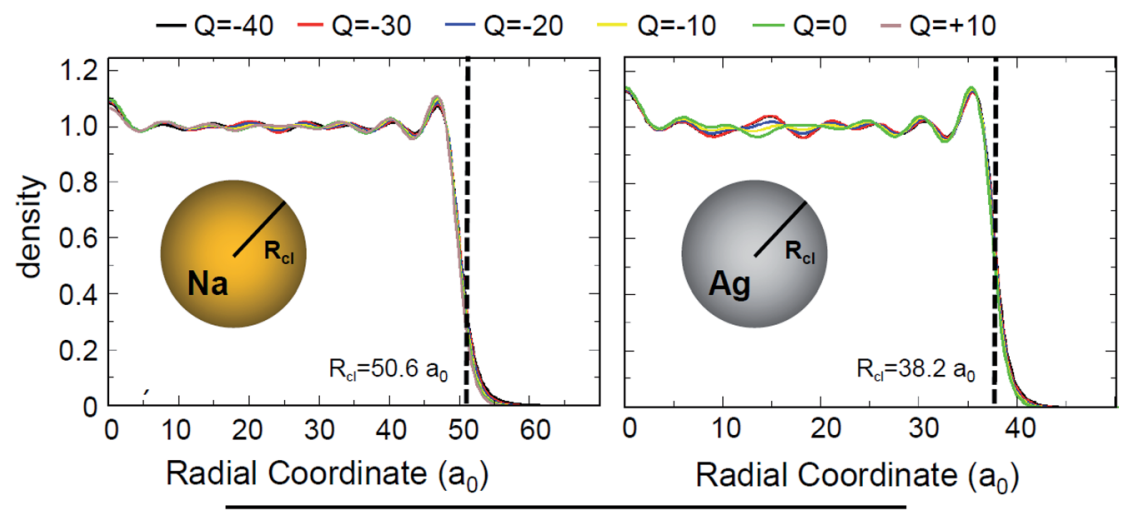

Distribution of charge excess in a particle
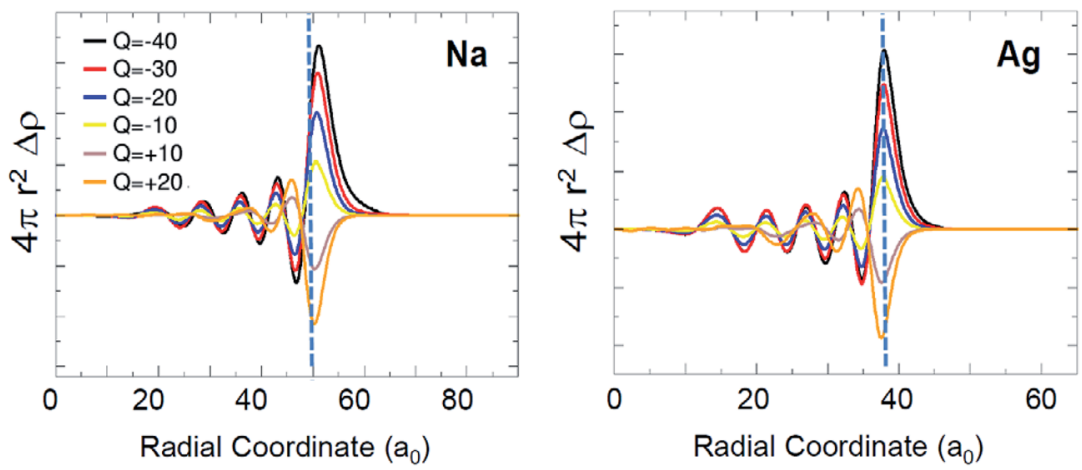

\section{Optical Spectra}
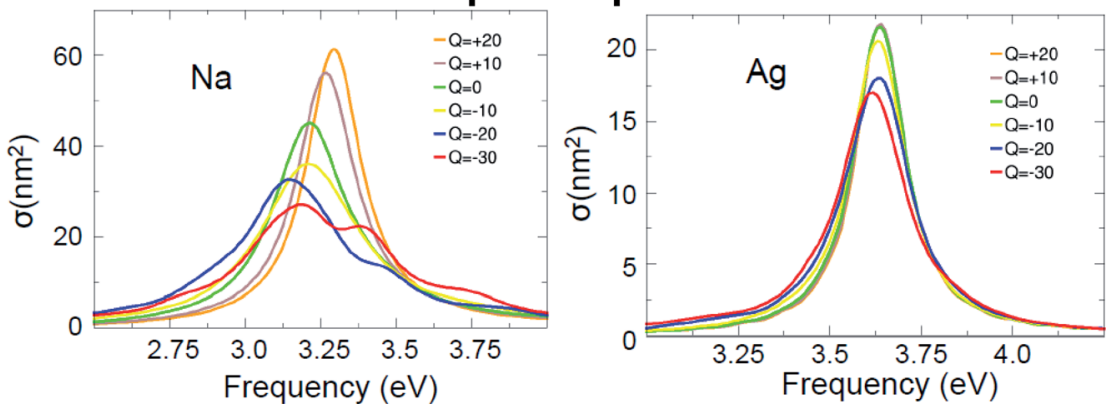

Fig. 9 Data on electron charge density, charge excess distribution and optical response of metallic clusters as a function of the clusters charge $Q$, based on material published in ref. 1 
Kei Murakoshi responded: The shift of the LSP energy depending on the electrochemical potential is also confirmed by the control experiment on the present system without dyes. The observation clearly proves that the change in the potential energy of the Fermi level in metal particles results in a shift of the LSPR energy. A linear shift in the peak energy of the extinction spectrum to higher energy is observed upon polarizing the electrochemical potential polarization to negative (closer to the vacuum level). Typical values of the shift are about 20-50 $\mathrm{meV}$ (a few tens of $\mathrm{nm}$ in wavelength) per $0.8 \mathrm{~V}$ electrochemical potential change as in the previously documented results. It is noteworthy that the shift is confirmed at the potential region where no electrochemical reactions proceed. This potential region is called the "double layer region" in the field of electrochemistry, where a net Faraday current is not observed. Just the Fermi level is linearly changed with the electrochemical potential polarization. We may carefully consider the difference between the simple charging model of yours and the electrochemical model in which the metal bulk density of states also shifts relative to the vacuum level with the electrochemical polarization. If the energy pinning of the metal surface could remain, a potential gradient at the metal surface may contribute to the change in the LSP energy.

Zhong-Qun Tian asked: It's true that the charge distribution and chemical and physical properties of the interface are strongly influenced by the environment, especially the dielectric constant of the electrolyte solution. When we change the aqueous electrolyte solution to a conductive ionic liquid, the applied potential window can be extended significantly from about $1.2 \mathrm{~V}$ to $3 \mathrm{~V}$ or more. We can apply a very negative electrode potential such as $-2.0 \mathrm{~V}$ ( $v s$. SCE), and thus the Jellium electrons will be forced to spill over from the metal electrode to solution. The liquid side will be filled with many solvated electrons thus creating a unique interface structure. An unexpectedly strong SERS signal of the ionic liquid can be obtained, five to ten times stronger than that at about $-1.0 \mathrm{~V}$.

Javier Aizpurua replied: This is a very interesting observation, and somewhat corroborates my comment on the paper of $\mathrm{H}$. Minamimoto et al. presented in this Faraday Discussion. ${ }^{1}$ In that comment I pointed out the importance of the interface electronic distribution and the whole environment in explaining plasmonic effects such as shifts and the corresponding local field enhancements, rather than simply relying on explanations based on the change of the bulk metallic electron density, which is usually not modified (see my comment to the paper above). The results you bring up here precisely point towards dramatic effects driven by the electronic structure of the metallic-liquid interface, when one changes the electrochemical environment from an aqueous electrolyte solution to a conductive ionic liquid, for example. The new electronic interface structure apparently provides a substantial enhancement of the Raman signal. As a theoretician, this is the kind of situation I would like to tackle: a full firstprinciples calculation of the optical response of a metallic structure where we can properly account for the distribution of the electronic density at the interface, including any effect of polarization or charging of the external electrochemical medium. In other words, a proper ab initio study of the metallic interface plus the external liquids. Both the ground state as well as the excited state (the plasmon) determine the final optical response and thus the enhancement. As you know 
yourself, to include at the $a b$ initio level the interfaces and the environment to calculate the optical response in realistic metallic structures of "large" size is really challenging and out of the reach of current computational methods. Your experimental results are a good example of the importance of considering the electronic "structure" of the interface in complex situations to accurately determine the optical response and the enhancement properties. More research on this aspect at the fundamental level is thus needed.

1 H. Minamimoto, F. Kato, F. Nagasawa, M. Takase and K. Murakoshi, Faraday Discuss., 2017, DOI: 10.1039/c7fd00126f.

Alex Keeler communicated: In Fig. 5 of your manuscript you have SERS and fluorescence intensities versus time where the potential is stepped from $0.0 \mathrm{~V}$ to $-0.60 \mathrm{~V},-0.70 \mathrm{~V}$ and $-0.80 \mathrm{~V} v \mathrm{~s}$. $\mathrm{Ag} / \mathrm{AgCl}$. Could you explain why you have a jump in both the SERS and fluorescence intensities in (b) at the same time that the potential is stepped to $-0.70 \mathrm{~V}$, before the intensities then reduce? In addition, do you have any explanation as to why this is not observed in either (a) or (c) when the potentials are stepped to -0.60 and $-0.80 \mathrm{~V}$ ?

Kei Murakoshi communicated in reply: Jumps both in the Raman and background intensities after the potential step to $-0.7 \mathrm{~V}$ should be due to the increment in the coupling strength. The LSP energy at $-0.7 \mathrm{~V}$ is closer to that of the dye exciton than those at -0.6 and $-0.8 \mathrm{~V}$. We confirmed the dependence of metal nanostructures with distinct LSP energies on the Raman and background intensities. The electrochemical potential where the jump-up behavior is observed is varied by the metal nanostructures showing distinct coupling strengths.

Duncan Graham opened a discussion of the paper by Fabrizio Giorgis: Have you tried a different excitation frequency with Cy5? $633 \mathrm{~nm}$ will bring in a strong term from the molecular resonance. In our paper in $2007^{1}$ we show the detection limits for combinations of excitation and labelled DNA using SERS and the molecular resonance term is very important for sensitivity.

1 R. J. Stokes, A. Macaskill, P. J. Lundahl, W. E. Smith, K. Faulds and D. Graham, Small, 2007, 3, 593-1601.

Fabrizio Giorgis responded: I agree with Duncan Graham on the importance of the excitation wavelength on the LOD taking into account the reporter optical absorbance. In our case we checked the performances of the SERS substrates using a laser line at $514.5 \mathrm{~nm}$, testing both Cy5 and Cy3 as Raman labels. This has been done in order to verify the influence of the molecular resonance on the detection limit. Actually, the assay with Cy3 resonantly excited showed a LOD two orders of magnitude lower than the assay with Cy5 (working in the electronic offresonance condition).

Duncan Graham asked: microRNA is often complexed with proteins and other molecules. How did you get your data, in terms of extractions, treatments etc.? Was there any compromise in terms of hybridisation efficiencies between the synthetic model and cells? 
Fabrizio Giorgis replied: miRNAs in real samples are often bound to proteins (e.g. the Argonaute protein family) or enclosed in lipid vesicles and during the extraction, or in the following treatment steps, bias can be introduced, reducing the amount of target in the extract. In our case, we used two different RNA extraction kits, and the miR-222 in the sample was quantified by Reverse Transcription quantitative Polymerase Chain Reaction (RTqPCR). We also checked the amount of target in the extracts by ELISA and SERS, but we didn't check it with other methods. Anyway, all the samples were treated with the same protocol, so if biases occur, they should be reasonably applied to all the samples in the same way. The only compromise between the analysis of the synthetic model and cell extracts was related to the ionic strength of the solution. The ionic strength is a fundamental parameter during hybridization, as stated in the paper. Then, in order to fix the ionic strength to the optimal value for hybridization, the cell extracts were diluted $1: 2$ in SSC $10 \times$ to a final concentration of $5 \times$.

Jürgen Popp commented: The overall goal of this paper is to create an assay. When using SERS for this approach, you will need a very reproducible and reliable SERS surface. How reliable is your approach from batch to batch and how many batches have been used? How do you test the enhancement factor of a new batch before doing the SERS experiment? Is one sample one batch? Is the reproducibility comparable to a fluorescent assay? Can it compete?

Fabrizio Giorgis answered: The results shown concern SERS substrates of a different batch for each analysis performed with a specific analyte concentration. The SERS intensities reported in the calibration curves are averages performed on 100 points for each substrate after a Raman mapping, and the error bars correspond to the standard deviations. We checked the reproducibility of our SERS-active substrates in terms of the inter- and intra-substrate standard deviation of the SERS signal intensity using 4-mercaptobenzoic acid as probe molecule, following an approach described in ref. 1. Such a procedure yielded a SD lower than $10 \%$ for both inter- and intrasubstrate analysis (10-15 substrate replicas have been used for each analyte concentration).

We did not check the reproducibility of our approach for analyzing the fluorescence signal, but with regard to stability, we can assert that the SERS signal is surely more stable than the fluorescence one (i.e. the Raman signal is not affected by fast quenching under light irradiation).

1 C. Novara, S. Dalla Marta, A. Virga, A. Lamberti, A. Angelini, A. Chiadò, P. Rivolo, F. Geobaldo, V. Sergo, A. Bonifacio and F. Giorgis, J. Phys. Chem. C, 2016, 120, 1694616953.

Joshua Edel asked: The Raman reporter distance is rather far from the surface (approximately 6-7 nm). Have you tried systems where you can get the reporter closer without affecting the binding efficiency (e.g. due to steric interactions)?

Fabrizio Giorgis answered: We are now investigating different configurations to reduce the distance of the Raman reporter from the surface, in order to increase the SERS enhancement, decreasing the fluorescence background. Our 
strategy relies on labelling the oligonucleotides closer to the SERS substrate, which is at the $3^{\prime}$ or at the $5^{\prime}$ terminal of the miR-222 or half 2 probe, for the onestep or two-step assay, respectively. Some steric hindrance could be expected in these configurations, but the experimental results demonstrate that almost a 4fold enhancement of the Raman signal is observed when the reporter is closest to the surface (with the one-step assay), hence the reduction of the binding efficiency should be a negligible effect.

Carin Lightner asked: Have you considered using multivariate analysis?

Fabrizio Giorgis responded: In our work, the study concerned SERS spectra of a single Raman reporter, thus our analysis dealt with linear regressions on the calibration curves aimed to extract the LODs. Anyway, I agree that multivariate analysis is a powerful tool, and necessary for a reliable multiplexed approach (i.e. the analysis of several miRNA sequences).

Marjorie Willner asked: Were the SERS measurements for the bioassay collected when the samples were wet or dry? If they were done on dried samples, were any drying effects observed? Were spectra collected through air or using a water immersion lens?

Fabrizio Giorgis responded: The bioassays were performed on the SERS substrates integrated in the optofluidic chip in a suitable liquid environment devoted to promoting the biorecognition mechanism. After that, the substrate surfaces have been gently dried under $\mathrm{N}_{2}$ flux and then measured through air by means of a standard objective (in a backscattering configuration). This choice was done with the aim to retain, during spectra collection in the mapping mode, the hybridization complexes formed after a specific incubation time. Thus, any kind of reversible effect in the strand coupling due to association-dissociation kinetics still occurring in a liquid environment should be avoided at the equilibrium conditions.

Howbeer Muhamadali said: In the paper, the quantification of the target molecule (miR-222) in cell extracts has been carried out using the calibration curves that were generated using pure molecule solutions. Wouldn't it be more appropriate to use the cell extracts of $\mathrm{H} 460$ cells to prepare the calibration curves? That way you will take several influencing factors into consideration, such as the complexity of the background solution and also the background concentration of your target molecule (miR-222), which according to your ELISA results is at a considerable level.

Fabrizio Giorgis replied: The quantification of miR-222 in the cell extracts has been carried out using the calibration curves that were generated using pure molecule solutions. At the same time, we checked the interfering matrix effect given by real biological samples, as suggested. As stated in the "miR-222 detection in cell extracts" paragraph of the paper, "to check the detection of miR-222 in real samples, the synthetic miR-222 was diluted in the total RNA extract of H460 NSCLC cells in a range of concentrations from $250 \mathrm{nM}$ to $0.5 \mathrm{nM}$ ". ${ }^{1}$ The results were compared with the calibration curve obtained using the synthetic miR-222 
diluted in SSC 4x-SDS (Fig. 6b and c of ref. 1), pointing out that the average spectra acquired by SERS mapping are very similar in both conditions (see an example in Fig. 10, showing a comparison of SERS spectra where the same concentration of miR-222 was incubated in buffer and in the cell extract) and the two calibration curves overlap within the error bars. Then, the calibration curve obtained using the synthetic miR-222 diluted in SSC 4x-SDS was exploited for the quantitation of the target in real samples.

1 C. Novara, A. Chiadò, N. Paccotti, S. Catuogno, C. L. Esposito, G. Condorelli, V. De Franciscis, F. Geobaldo, P. Rivolo and F. Giorgis, Faraday Discuss., 2017, DOI: 10.1039/ c7fd00140a.

\section{miR-222 $50 \mathrm{nM}$}

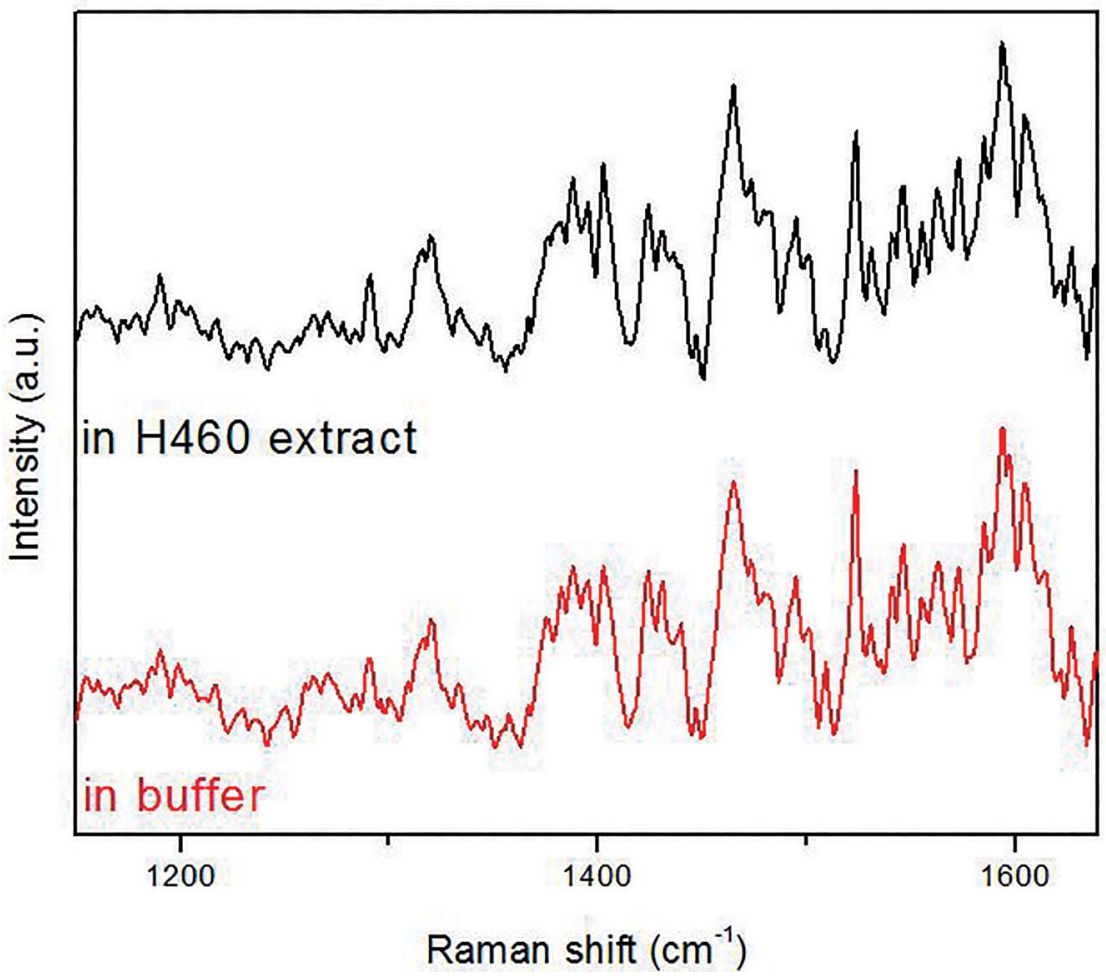

Fig. 10 SERS spectra of assays where the same concentration of miR-222 was incubated in buffer and in the cell extract.

Joshua Edel asked: Although I very much like the strategy being used, I wonder what the advantages are over using fluorescence? Such assays have been around for some time and can be based around very simple instrumental platforms. Do you have a long-term vision in terms of truly taking on the advantages of SERS?

Fabrizio Giorgis replied: The advantages of SERS $v s$. fluorescence detection concern the possibility of a multiplexed approach with a considerable number of analytes, exploiting the narrow Raman bands (related to the biomolecule or to the 
applied reporter) with respect to the broad fluorescence ones. Moreover, fluorescence spectra are characterized by a single band (usually, in standard detection methods they are even analyzed only on a specific wavelength) whilst Raman/ SERS spectra are distinguished by many resonances which can be analyzed with a multivariate approach in order to improve the reliability of the analytical measurement. In this sense, in particular for the multiplexed analysis needed for miRNA profiling, SERS platforms show significant advantages over the standard optical methods.

Xinping Wang asked: microRNAs are sensitive to RNase and could be digested easily. There are multiple steps to construct the SERS substrate in the assay. RNase could simply be involved in such processes. Have you taken some precautions against damage of the structure of the microRNAs? The structure stability of the analytes will directly relate to the detection limit of the assay.

Fabrizio Giorgis responded: RNase can effectively digest miRNAs and RNA, reducing the effectiveness of the analysis. However, even if RNase is highly stable in different conditions, a contamination is very unlikely to occur during the fabrication of the SERS substrates because of the reagents involved (e.g. HF, ethanol, etc.). At the same time, there are several steps during the whole sensing protocol that could lead to RNase contamination, but we tried to reduce it to a minimum. First of all, the workbench, the laminar-flow hood and all the work areas were cleaned with specific products (e.g. RNaseZAP). Finally, all the buffer solutions were prepared with DEPC-treated sterile water.

Ian Bruzas asked: You have mentioned a large fluorescence background, is any of this due to surface enhanced fluorescence? Have you compared the LOD for fluorescence $v s$. SERS? How does the raw LOD compare?

Fabrizio Giorgis answered: The tests for the effect of the Raman reporter position along the oligonucleotide strand (see the question from Joshua Edel) on the SERS enhancement have also shown that the fluorescence background is strongly dependent on the distance from the surface. When the reporter is less than $1 \mathrm{~nm}$ away from the nanoparticles, the background is the lowest, while it increases for intermediate distances (about $3.5 \mathrm{~nm}$ ) and becomes very large for the highest probed distances $(7 \mathrm{~nm})$. Despite this experimental finding, we cannot state with certainty if we are observing surface enhanced fluorescence (in the spectra analyzed in the paper), as more data are needed to distinguish the variation of the emitter quantum yield and the excitation rate, which will decrease/ increase respectively when the emitter approaches the nanoparticle, as is well known for metal-enhanced fluorescence processes. Actually, we have not calculated the LOD for the fluorescence detection with our sensing platform.

Malama Chisanga communicated: miRNA and small interfering RNAs (siRNAs of the interference pathway of RNA) have closely related sequences, and hence resemble each other. In a real biological complex sample mixture where miRNAs of different origins interact with each other, and with siRNAs, would this SERS technique be sensitive enough to identify and discriminate between miRNAs and siRNAs accurately? Would it be able to detect interactions as well? 
Fabrizio Giorgis communicated in response: miRNA and siRNA are characterized by a very high sequence similarity, hence the discrimination between them can be not trivial. Actually, both are generated by the Dicer/RISC complex and the major difference between siRNAs and miRNAs is that the former are highly specific with only one mRNA target, whereas the latter have multiple targets, as reported elsewhere. ${ }^{1}$ In our paper, we used a perfect complementary probe to detect miR-222, and even if we tested the selectivity of the bioassay by checking the assay with other miRNA sequences with positive results, we never tried SERS detection on synthetic siRNA or single-base mismatch sequences. For sure this will be an interesting future check aimed at understanding the potential of our approach.

1 J. K. W. Lam, M. Y. T. Chow, Y. Zhang and S. W. S. Leung, Mol. Ther.-Nucleic Acids, 2015, 4, e252.

Holly Fleming communicated: What size, or size range, of nanoparticles were synthesized? Are the nanoparticles in a stable arrangement, and are they affected by the functionalisation, washing and drying steps?

Fabrizio Giorgis communicated in response: Our SERS substrates consist of silver nanoparticles with an average size around $40 \mathrm{~nm}$, grown on mesoporous silicon membranes attached to PDMS slices. This system has been carefully checked in terms of efficiency and stability by Raman spectroscopy and electron microscopy after the several steps of the biological protocols. The results demonstrate good stability with respect to other analyzed metal-dielectric nanostructures synthesized in our lab. ${ }^{1}$

1 A. Chiadò, C. Novara, A. Lamberti, F. Geobaldo, F. Giorgis and P. Rivolo, Anal. Chem., 2016, 88, 9554-9563. 